University of South Carolina

Scholar Commons

$5-2-2008$

\title{
An Optimal-Order Error Estimate for a Family of ELLAM-MFEM Approximations to Porous Medium Flow
}

Hong Wang

University of South Carolina - Columbia, wang@math.sc.edu

Follow this and additional works at: https://scholarcommons.sc.edu/math_facpub

Part of the Mathematics Commons

Publication Info

Published in Siam Journal of Numerical Analysis, Volume 46, Issue 4, 2008, pages 2133-2152.

(C) SIAM Journal On Numerical Analysis 2008, Society for Industrial and Applied Mathematics Wang, H. (2008). An Optimal-Order Error Estimate for a Family of ELLAM-MFEM Approximations to Porous Medium Flow. SIAM Journal On Numerical Analysis, 46(4), 2133-2152. doi: 10.1137/ s0036142903428281

This Article is brought to you by the Mathematics, Department of at Scholar Commons. It has been accepted for inclusion in Faculty Publications by an authorized administrator of Scholar Commons. For more information, please contact digres@mailbox.sc.edu. 


\title{
AN OPTIMAL-ORDER ERROR ESTIMATE FOR A FAMILY OF ELLAM-MFEM APPROXIMATIONS TO POROUS MEDIUM FLOW*
}

\author{
HONG WANG ${ }^{\dagger}$
}

\begin{abstract}
Mathematical models used to describe porous medium flow lead to coupled systems of time-dependent nonlinear partial differential equations, which present serious mathematical and numerical difficulties. Standard methods tend to generate numerical solutions with nonphysical oscillations or numerical dispersion along with spurious grid-orientation effect. The ELLAM-MFEM time-stepping procedure, in which an Eulerian-Lagrangian localized adjoint method (ELLAM) is used to solve the transport equation and a mixed finite element method (MFEM) is used for the pressure equation, simulates porous medium flow accurately even if large spatial grids and time steps are used. In this paper we prove an optimal-order error estimate for a family of ELLAM-MFEM approximations.
\end{abstract}

Key words. advection-diffusion equations, characteristic methods, convergence analysis, error estimates, Eulerian-Lagrangian methods, numerical analysis, porous medium flow, subsurface flow

AMS subject classifications. 65M12, 65M15, 65M25, 65M60, 65Z05, 76M10, 76S05

DOI. $10.1137 / \mathrm{S} 0036142903428281$

1. Introduction. Mathematical models used to describe porous medium flow processes in petroleum reservoir simulation, groundwater contaminant transport, and other applications lead to coupled systems of time-dependent nonlinear partial differential equations (PDEs) $[2,11]$. Due to the advection-diffusion feature of the transport PDEs, the nonlinearity and couplings of the PDEs in the systems, and the strong effect of sources and sinks, these systems admit solutions with moving steep fronts and complicated structures. Consequently, these problems present serious mathematical and numerical difficulties. Standard finite difference or finite element methods (FDMs and FEMs, respectively) tend to generate solutions with nonphysical oscillations. Upwind methods are often used to stabilize the numerical approximations, but they often produce numerical dispersion and the grid-orientation effect [11]. Extensive research has been carried out on the development of improved methods for advection-diffusion PDEs $[16,18,21]$.

An FEM-MFEM time-stepping procedure was presented and analyzed in $[8,9]$. In the procedure, a mixed finite element method (MFEM) scheme $[4,5,20]$ was used to solve the pressure PDE, and an FEM was used to solve the transport PDE. MFEM schemes generate an accurate approximation to the Darcy velocity, which is required for accurate approximation to the concentration because advection and diffusion dispersion in the transport PDE are governed by Darcy velocity. MFEMs minimize the numerical difficulties occurring in FDMs or FEMs caused by differentiation of the pressure and then multiplication by rough coefficients [22]. Subsequently, an MMOCMFEM time-stepping procedure was proposed and analyzed in $[12,13]$, in which the modified method of characteristics (MMOC) [10] was used to replace the FEM scheme in [9]. The MMOC symmetrizes and stabilizes the transport PDE, greatly reduces temporal errors, and so allows for large time steps in a simulation without loss of

\footnotetext{
${ }^{*}$ Received by the editors May 15, 2003; accepted for publication (in revised form) October 25, 2007; published electronically May 2, 2008.

http://www.siam.org/journals/sinum/46-4/42828.html

${ }^{\dagger}$ Department of Mathematics, University of South Carolina, Columbia, SC 29208 (hwang@math. sc.edu).
} 
accuracy. However, the MMOC fails to conserve mass and has difficulties in handling general boundary conditions.

The Eulerian-Lagrangian localized adjoint method (ELLAM) framework [6, 21] provides a systematic approach to solve transient advection-diffusion PDEs with general boundary conditions in a mass-conservative manner, while maintaining accuracy and efficiency of Eulerian-Lagrangian methods. Numerical experiments show that the ELLAM is very competitive in the context of model transport PDEs [26, 28] and miscible porous medium flow $[3,17,30,32]$. Nevertheless, corresponding theoretical analysis falls far behind. To date, error estimates for the ELLAM and related schemes have been proved in the context of linear advection-dominated transport PDEs $[1,14,15,24,25,27,29,31]$. This is partly due to the fact that the analyses are more complex than those for the MMOC and related methods [10, 29, 21]. In this paper we derive an optimal-order error estimate for an ELLAM-MFEM approximation to the coupled system of miscible porous medium flow.

The rest of the paper is organized as follows: In section 2 we review the mathematical model. In section 3 we describe the ELLAM-MFEM time-stepping procedure. In section 4 we present known theoretical results used in the analysis. In section 5 we prove an optimal-order error estimate for the ELLAM-MFEM time-stepping procedure. In section 6 we prove several lemmas used in section 5 .

2. Mathematical model and notation. We present a mathematical model for porous medium flow and introduce the functional spaces used in this paper.

2.1. Mathematical model. Let $c(\mathbf{x}, t)$ be the concentration of an invading fluid or a concerned solute/solvent, and let $p(\mathbf{x}, t)$ and $\mathbf{u}(\mathbf{x}, t)$ be the pressure and Darcy velocity of the fluid mixture, respectively. The mass conservation for the fluid mixture incorporated with the incompressibility condition, Darcy's law, and the mass conservation for the invading fluid lead to the following system of PDEs [2, 11]:

$$
\begin{array}{rlrl}
\nabla \cdot \mathbf{u} & =q, \quad \mathbf{u}=-\frac{\mathbf{K}}{\mu(c)}(\nabla p-\rho g \nabla d), & & \mathbf{x} \in \Omega, \quad t \in[0, T], \\
\phi \frac{\partial c}{\partial t}+\nabla \cdot(\mathbf{u} c-\mathbf{D}(\mathbf{x}, \mathbf{u}) \nabla c)=\bar{c} q, & & \mathbf{x} \in \Omega, \quad t \in[0, T], \\
\mathbf{u} \cdot \mathbf{n} \quad=(\mathbf{D} \nabla c) \cdot \mathbf{n}=0, & & \mathbf{x} \in \partial \Omega, \quad t \in[0, T], \\
c(\mathbf{x}, 0) & =c_{0}(\mathbf{x}), & & \mathbf{x} \in \Omega .
\end{array}
$$

We assume that the medium is homogeneous vertically and take $\Omega \subset \mathbb{R}^{2}$ with a nonuniform local elevation. $\phi(\mathbf{x})$ and $\mathbf{K}(\mathbf{x})$ are the porosity and the permeability tensor of the medium, respectively, $\mu(c)$ and $\rho$ are the viscosity and the density of the fluid mixture, respectively, $g$ is the gravitational acceleration, $d(\mathbf{x})$ is the reservoir depth, and $q(\mathbf{x}, t)$ is the source and sink term. $\mathbf{D}(\mathbf{x}, \mathbf{u})=\phi(\mathbf{x}) d_{m} \mathbf{I}+d_{t}|\mathbf{u}|+\left(d_{l}-\right.$ $\left.d_{t}\right)\left(u_{i} u_{j}\right)_{i, j=1}^{2} /|\mathbf{u}|$ is the diffusion-dispersion tensor, with $d_{m}, d_{t}$, and $d_{l}$ being the molecular diffusion and the transverse and longitudinal dispersivities, respectively, and $\mathbf{I}$ is the identity tensor. $\bar{c}(\mathbf{x}, t)$ is specified at sources and $\bar{c}(\mathbf{x}, t)=c(\mathbf{x}, t)$ at sinks. $c_{0}(\mathbf{x})$ is the initial concentration.

The combination of the first equations in (2.1) and (2.3) implies that $q$ has mean 0 . In addition, (2.1) with the noflow boundary condition (2.3) can determine the pressure $p(\mathbf{x}, t)$ only up to an additive constant for all the time $t \in[0, T]$. But this indeterminacy is of no consequence since $\mathbf{u}$ is uniquely determined by Darcy's law, and only $\mathbf{u}(\operatorname{not} p)$ is needed in (2.2). 
2.2. Notation. Let $W_{q}^{m}(\Omega)$ be the Sobolev spaces consisting of functions whose derivatives up to order- $m$ are $q$ th integrable on $\Omega$, and $H^{m}(\Omega):=W_{2}^{m}(\Omega)$. Let $L_{0}^{2}(\Omega)$ be the subspace of $L^{2}(\Omega)$ with mean 0 . We introduce vector-valued Sobolev spaces

$$
\begin{aligned}
H^{m}(\operatorname{div} ; \Omega) & :=\left\{\mathbf{f}(\mathbf{x})=\left(f_{1}, f_{2}\right): f_{1}, f_{2}, \nabla \cdot \mathbf{f} \in H^{m}(\Omega)\right\} \\
\|\mathbf{f}\|_{H^{m}(\operatorname{div} ; \Omega)} & :=\left(\left\|f_{1}\right\|_{H^{m}(\Omega)}^{2}+\left\|f_{2}\right\|_{H^{m}(\Omega)}^{2}+\|\nabla \cdot \mathbf{f}\|_{H^{m}(\Omega)}^{2}\right)^{1 / 2} \\
H_{0}(\operatorname{div} ; \Omega) & :=\left\{\mathbf{f}(\mathbf{x}) \in H^{0}(\operatorname{div} ; \Omega): \mathbf{f}(\mathbf{x}) \cdot \mathbf{n}(\mathbf{x})=0, \mathbf{x} \in \partial \Omega\right\}
\end{aligned}
$$

We drop $\Omega$ in these notations when it is clear from the context.

For any Banach space $X$, we introduce Sobolev spaces involving time

$$
\begin{aligned}
W_{q}^{m}\left(t_{1}, t_{2} ; X\right) & :=\left\{f(\mathbf{x}, t):\left\|\frac{\partial^{\alpha} f}{\partial t^{\alpha}}(\cdot, t)\right\|_{X} \in L^{q}\left(t_{1}, t_{2}\right), 0 \leq \alpha \leq m, 1 \leq q \leq \infty\right\}, \\
\|f\|_{W_{q}^{m}\left(t_{1}, t_{2} ; X\right)}:= & \begin{cases}\left(\sum_{\alpha=0}^{m} \int_{t_{1}}^{t_{2}}\left\|\frac{\partial^{\alpha} f}{\partial t^{\alpha}}(\cdot, t)\right\|_{X}^{q} d t\right)^{1 / q}, & 1 \leq q<\infty \\
\max _{0 \leq \alpha \leq m} \operatorname{esssup}_{t \in\left(t_{1}, t_{2}\right)}\left\|\frac{\partial^{\alpha} f}{\partial t^{\alpha}}(\cdot, t)\right\|_{X}, & q=\infty .\end{cases}
\end{aligned}
$$

We also define the discrete norms

$$
\begin{aligned}
\|f\|_{\hat{L}_{c}^{\infty}(0, T ; X)} & :=\max _{0 \leq n \leq N}\left\|f\left(\mathbf{x}, t_{n}^{c}\right)\right\|_{X}, \quad\|f\|_{\hat{L}_{p}^{\infty}(0, T ; X)}:=\max _{0 \leq m \leq M}\left\|f\left(\mathbf{x}, t_{m}^{p}\right)\right\|_{X}, \\
\|f\|_{\hat{L}_{c}^{2}(0, T ; X)} & :=\left(\sum_{n=0}^{N}\left\|f\left(\mathbf{x}, t_{n}^{c}\right)\right\|_{X}^{2} \Delta t_{n}^{c}\right)^{1 / 2},
\end{aligned}
$$

with $t_{n}^{c}$ and $t_{m}^{p}$ being defined in (3.1) and (3.11), respectively. If $\left(t_{1}, t_{2}\right)=(0, T)$, we drop it from these notations.

In this paper we use $\varepsilon$ to denote an arbitrarily small positive number, $A_{i}, K_{i}$, and $Q_{i}$ to denote fixed positive constants, and $Q$ to denote a generic positive constant that could assume different values at different occurrences. All of these constants are independent of spatial and temporal grid parameters.

3. An ELLAM-MFEM time-stepping procedure. In this procedure an ELLAM scheme is used to solve the transport PDE (2.2), and an MFEM scheme is used for the pressure system (2.1).

3.1. An ELLAM formulation for the transport equation. We define a temporal partition on the interval $[0, T]$ by

$$
0=: t_{0}^{c}<t_{1}^{c}<\cdots<t_{n}^{c}<\cdots<t_{N-1}^{c}<t_{N}^{c}:=T,
$$

with $\Delta t_{n}^{c}:=t_{n}^{c}-t_{n-1}^{c}$ and $\Delta t_{c}:=\max _{1 \leq n \leq N} \Delta t_{n}^{c}$.

By multiplying (2.2) by any space-time test functions $z$ that are continuous and piecewise smooth, vanish outside the space-time strip $\Omega \times\left(t_{n-1}^{c}, t_{n}^{c}\right]$, and are discon- 
tinuous in time at time $t_{n-1}^{c}$, we obtain a weak formulation for (2.2)

$$
\begin{gathered}
\int_{\Omega} \phi(\mathbf{x}) c\left(\mathbf{x}, t_{n}^{c}\right) z\left(\mathbf{x}, t_{n}^{c}\right) d \mathbf{x}+\int_{t_{n-1}^{c}}^{t_{n}^{c}} \int_{\Omega} \nabla z(\mathbf{y}, \theta) \cdot \mathbf{D}(\mathbf{y}, \mathbf{u}(\mathbf{y}, \theta)) \nabla c(\mathbf{y}, \theta) d \mathbf{y} d \theta \\
-\int_{t_{n-1}^{c}}^{t_{n}^{c}} \int_{\Omega} c(\mathbf{y}, \theta)\left[\phi(\mathbf{y}) \frac{\partial z(\mathbf{y}, \theta)}{\partial \theta}+\mathbf{u}(\mathbf{y}, \theta) \cdot \nabla z(\mathbf{y}, \theta)\right] d \mathbf{y} d \theta \\
=\int_{\Omega} \phi(\mathbf{x}) c\left(\mathbf{x}, t_{n-1}^{c}\right) z\left(\mathbf{x}, t_{n-1}^{c,+}\right) d \mathbf{x}+\int_{t_{n-1}^{c}}^{t_{n}^{c}} \int_{\Omega} \bar{c}(\mathbf{y}, \theta) q(\mathbf{y}, \theta) z(\mathbf{y}, \theta) d \mathbf{y} d \theta
\end{gathered}
$$

where $z\left(\mathbf{x}, t_{n-1}^{c,+}\right):=\lim _{t \rightarrow t_{n-1}^{c}}, t>t_{n-1}^{c} z(\mathbf{x}, t)$ accounts for the discontinuity of $z(\mathbf{x}, t)$ in time at time $t_{n-1}^{c}$. We replace the dummy variables $\mathbf{x}$ and $t$ in the space-time integrals in (3.2) by $\mathbf{y}$ and $\theta$ and reserve $\mathbf{x}$ for the point in $\Omega$ at time $t_{n}^{c}$ or $t_{n-1}^{c}$.

In the ELLAM framework [6,21,30], the test functions in (3.2) are defined to be the solutions of the adjoint equation of the hyperbolic part of $(2.2)$

$$
\phi(\mathbf{y}) \frac{\partial z(\mathbf{y}, \theta)}{\partial \theta}+\mathbf{u}(\mathbf{y}, \theta) \cdot \nabla z(\mathbf{y}, \theta)=0, \quad \mathbf{y} \in \bar{\Omega}, \quad \theta \in\left[t_{n-1}^{c}, t_{n}^{c}\right]
$$

Thus, the last term on the left-hand side of (3.2) vanishes. Once the test functions $z\left(\mathbf{x}, t_{n}^{c}\right)$ are specified in $\Omega$, they are determined in the space-time strip $\Omega \times\left(t_{n-1}^{c}, t_{n}^{c}\right]$ by constant extension along the characteristics $\mathbf{y}=\mathbf{r}\left(\theta ; \mathbf{x}, t_{n}^{c}\right)$ defined by

$$
\frac{d \mathbf{r}}{d \theta}=\frac{\mathbf{u}(\mathbf{r}, \theta)}{\phi(\mathbf{r})} \quad \text { and }\left.\quad \mathbf{r}(\theta ; \overline{\mathbf{x}}, \bar{t})\right|_{\theta=\bar{t}}=\overline{\mathbf{x}}
$$

Evaluating the source term in (3.2) by the Euler formula at time $t_{n}^{c}$ yields

$$
\begin{aligned}
& \int_{t_{n-1}^{c}}^{t_{n}^{c}} \int_{\Omega} \bar{c}(\mathbf{y}, \theta) q(\mathbf{y}, \theta) z(\mathbf{y}, \theta) d \mathbf{y} d \theta \\
& \quad=\int_{\Omega} \int_{t_{n-1}^{c}}^{t_{n}^{c}} \bar{c}\left(\mathbf{r}\left(\theta ; \mathbf{x}, t_{n}^{c}\right), \theta\right) q\left(\mathbf{r}\left(\theta ; \mathbf{x}, t_{n}^{c}\right), \theta\right) z\left(\mathbf{x}, t_{n}^{c}\right) \operatorname{det}\left(\frac{\partial \mathbf{r}\left(\theta ; \mathbf{x}, t_{n}^{c}\right)}{\partial \mathbf{x}}\right) d \theta d \mathbf{x} \\
& \quad=\Delta t_{n}^{c} \int_{\Omega} \bar{c}\left(\mathbf{x}, t_{n}^{c}\right) q\left(\mathbf{x}, t_{n}^{c}\right) z\left(\mathbf{x}, t_{n}^{c}\right) d \mathbf{x}+E_{q}(\bar{c}, z)
\end{aligned}
$$

where $E_{q}(\bar{c}, z)$ is the local truncation error term given by

$$
\begin{aligned}
E_{q}(\bar{c}, z): & =-\int_{\Omega} z\left(\mathbf{x}, t_{n}^{c}\right)\left\{\int _ { t _ { n - 1 } ^ { c } } ^ { t _ { n } ^ { c } } \left[\bar{c}\left(\mathbf{x}, t_{n}^{c}\right) q\left(\mathbf{x}, t_{n}^{c}\right)\right.\right. \\
& \left.\left.-\bar{c}\left(\mathbf{r}\left(\theta ; \mathbf{x}, t_{n}^{c}\right), \theta\right) q\left(\mathbf{r}\left(\theta ; \mathbf{x}, t_{n}^{c}\right), \theta\right) \operatorname{det}\left(\frac{\partial \mathbf{r}\left(\theta ; \mathbf{x}, t_{n}^{c}\right)}{\partial \mathbf{x}}\right)\right] d \theta\right\} d \mathbf{x} .
\end{aligned}
$$

In (3.5) we have implicitly used the fact that the traceback operator $\mathbf{r}$ is a diffeomorphism and the determinant is positive [10]. 
Likewise, we evaluate the diffusion-dispersion term in (3.2) to obtain

$$
\begin{aligned}
& \int_{t_{n-1}^{c}}^{t_{n}^{c}} \int_{\Omega} \nabla z(\mathbf{y}, \theta) \cdot \mathbf{D}(\mathbf{y}, \mathbf{u}(\mathbf{y}, \theta)) \nabla c(\mathbf{y}, \theta) d \mathbf{y} d \theta \\
& =\int_{\Omega} \int_{t_{n-1}^{c}}^{t_{n}^{c}} \nabla_{\mathbf{r}} z\left(\mathbf{x}, t_{n}^{c}\right) \cdot\left(\mathbf{D}\left(\mathbf{r}\left(\theta ; \mathbf{x}, t_{n}^{c}\right), \mathbf{u}\left(\mathbf{r}\left(\theta ; \mathbf{x}, t_{n}^{c}\right), \theta\right)\right)\right. \\
& \left.\quad \nabla c_{\mathbf{r}}\left(\mathbf{r}\left(\theta ; \mathbf{x}, t_{n}^{c}\right), \theta\right)\right) \operatorname{det}\left(\frac{\partial \mathbf{r}\left(\theta ; \mathbf{x}, t_{n}^{c}\right)}{\partial \mathbf{x}}\right) d \theta d \mathbf{x} \\
& =\int_{\Omega} \int_{t_{n-1}^{c}}^{t_{n}^{c}} \operatorname{det}\left(\frac{\partial \mathbf{r}\left(\theta ; \mathbf{x}, t_{n}^{c}\right)}{\partial \mathbf{x}}\right)\left(\frac{\partial \mathbf{r}\left(\theta ; \mathbf{x}, t_{n}^{c}\right)}{\partial \mathbf{x}}\right)^{-1} \nabla z\left(\mathbf{x}, t_{n}^{c}\right) \\
& \quad \cdot\left(\mathbf{D}\left(\mathbf{r}\left(\theta ; \mathbf{x}, t_{n}^{c}\right), \mathbf{u}\left(\mathbf{r}\left(\theta ; \mathbf{x}, t_{n}^{c}\right), \theta\right)\right) \nabla c_{\mathbf{r}}\left(\mathbf{r}\left(\theta ; \mathbf{x}, t_{n}^{c}\right), \theta\right)\right) d \theta d \mathbf{x} \\
& =\Delta t_{n}^{c} \int_{\Omega} \nabla z\left(\mathbf{x}, t_{n}^{c}\right) \cdot \mathbf{D}\left(\mathbf{x}, \mathbf{u}\left(\mathbf{x}, t_{n}^{c}\right)\right) \nabla c\left(\mathbf{x}, t_{n}^{c}\right) d \mathbf{x}+E_{\mathbf{D}}(c, z) .
\end{aligned}
$$

The subscript $\mathbf{r}$ emphasizes that the gradient is taken with respect to $\mathbf{r}$. The local truncation error $E_{\mathbf{D}}(c, z)$ is given by

$$
\begin{aligned}
& E_{\mathbf{D}}(c, z):= \int_{\Omega} \int_{t_{n-1}^{c}}^{t_{n}^{c}}\left(\operatorname{det}\left(\frac{\partial \mathbf{r}\left(\theta ; \mathbf{x}, t_{n}^{c}\right)}{\partial \mathbf{x}}\right)\left(\frac{\partial \mathbf{r}\left(\theta ; \mathbf{x}, t_{n}^{c}\right)}{\partial \mathbf{x}}\right)^{-1}-\mathbf{I}\right) \nabla z\left(\mathbf{x}, t_{n}^{c}\right) \\
& \cdot\left(\mathbf{D}\left(\mathbf{r}\left(\theta ; \mathbf{x}, t_{n}^{c}\right), \mathbf{u}\left(\mathbf{r}\left(\theta ; \mathbf{x}, t_{n}^{c}\right), \theta\right)\right) \nabla c\left(\mathbf{r}\left(\theta ; \mathbf{x}, t_{n}^{c}\right), \theta\right)\right) d \theta d \mathbf{x} \\
&-\int_{\Omega} \int_{t_{n-1}^{c}}^{t_{n}^{c}} \nabla z\left(\mathbf{x}, t_{n}\right) \cdot\left(\mathbf{D}\left(\mathbf{x}, \mathbf{u}\left(\mathbf{x}, t_{n}^{c}\right)\right) \nabla c\left(\mathbf{x}, t_{n}^{c}\right)\right. \\
&\left.-\mathbf{D}\left(\mathbf{r}\left(\theta ; \mathbf{x}, t_{n}^{c}\right), \mathbf{u}\left(\mathbf{r}\left(\theta ; \mathbf{x}, t_{n}^{c}\right), \theta\right)\right) \nabla c\left(\mathbf{r}\left(\theta ; \mathbf{x}, t_{n}^{c}\right), \theta\right)\right) d \theta d \mathbf{x} .
\end{aligned}
$$

We substitute (3.5) and (3.7) into (3.2) to obtain an ELLAM reference equation for the transport PDE (2.2)

$$
\begin{aligned}
\int_{\Omega} \phi(\mathbf{x}) c\left(\mathbf{x}, t_{n}^{c}\right) z\left(\mathbf{x}, t_{n}^{c}\right) d \mathbf{x}+\Delta t_{n}^{c} \int_{\Omega} \nabla z\left(\mathbf{x}, t_{n}^{c}\right) \cdot \mathbf{D}\left(\mathbf{x}, \mathbf{u}\left(\mathbf{x}, t_{n}^{c}\right)\right) \nabla c\left(\mathbf{x}, t_{n}^{c}\right) d \mathbf{x} \\
=\int_{\Omega} \phi(\mathbf{x}) c\left(\mathbf{x}, t_{n-1}^{c}\right) z\left(\mathbf{x}, t_{n-1}^{c,+}\right) d \mathbf{x}+\Delta t_{n}^{c} \int_{\Omega} \bar{c}\left(\mathbf{x}, t_{n}^{c}\right) q\left(\mathbf{x}, t_{n}^{c}\right) z\left(\mathbf{x}, t_{n}^{c}\right) d \mathbf{x} \\
\quad-E_{\mathbf{D}}(c, z)+E_{q}(\bar{c}, z) .
\end{aligned}
$$

3.2. An MFEM formulation for the pressure and Darcy velocity. We multiply the second equation in $(2.1)$ by $\mu(c) \mathbf{K}^{-1}(\mathbf{x})$ and any test functions $\mathbf{v} \in$ $H_{0}($ div $; \Omega)$ and apply the divergence theorem to the $\nabla p$ term. We then multiply the first equation in (2.1) by any test functions $w(\mathbf{x}) \in L_{0}^{2}(\Omega)$ and integrate over $\Omega$. The system (2.1) is expressed as a time-parameterized saddle-point problem of finding a map $(\mathbf{u}(\mathbf{x}, t), p(\mathbf{x}, t)) \in H_{0}(\operatorname{div} ; \Omega) \times L_{0}^{2}(\Omega)$ such that

$$
\begin{aligned}
\int_{\Omega} \mu(c) \mathbf{K}^{-1} \mathbf{u} \cdot \mathbf{v} d \mathbf{x}-\int_{\Omega} p \nabla \cdot \mathbf{v} d \mathbf{x} & =\int_{\Omega} \rho g \nabla d \cdot \mathbf{v} d \mathbf{x}, \\
\int_{\Omega} w \nabla \cdot \mathbf{u} d \mathbf{x} & =\int_{\Omega} q(\mathbf{x}, t) w d \mathbf{x} \\
\forall(\mathbf{v}(\mathbf{x}), w(\mathbf{x})) \in H_{0}(\operatorname{div} ; \Omega) & \times L_{0}^{2}(\Omega), t \in[0, T] .
\end{aligned}
$$

Copyright $\odot$ by SIAM. Unauthorized reproduction of this article is prohibited. 
It was proved $[4,8]$ that problem $(3.10)$ has a unique solution $(\mathbf{u}(\mathbf{x}, t), p(\mathbf{x}, t)) \in$ $H_{0}(\operatorname{div} ; \Omega) \times L_{0}^{2}(\Omega)$ for all $t \in[0, T]$.

Let $V^{k} \subset H_{0}(\operatorname{div} ; \Omega)$ and $S_{p}^{k} \subset L_{0}^{2}(\Omega)$ be MFEM spaces of index $k \geq 0$ on a quasiuniform partition of $\Omega=\cup \Omega_{e}^{p}$ with the diameter $h_{p}[5,20]$. We define a temporal partition on the time interval $[0, T]$ for the pressure grid by

$$
0=: t_{0}^{p}<t_{1}^{p}<\cdots<t_{m}^{p}<\cdots<t_{M-1}^{p}<t_{M}^{p}:=T,
$$

with $\Delta t_{m}^{p}:=t_{m}^{p}-t_{m-1}^{p}$ and $\Delta t_{p}:=\max _{1 \leq m \leq M} \Delta t_{m}^{p}$.

Given a concentration approximation $c_{h}\left(\mathbf{x}, t_{m}^{p}\right)$ at time $t_{m}^{p}$, the MFEM scheme determines the velocity $\mathbf{u}_{h}\left(\mathbf{x}, t_{m}^{p}\right) \in V^{k}$ and the pressure $p_{h}\left(\mathbf{x}, t_{m}^{p}\right) \in S_{p}^{k}$ such that

$$
\begin{array}{cc}
\int_{\Omega} \mu\left(c_{h}\left(\mathbf{x}, t_{m}^{p}\right)\right) \mathbf{K}^{-1}(\mathbf{x}) \mathbf{u}_{h}\left(\mathbf{x}, t_{m}^{p}\right) \cdot \mathbf{v}_{h}(\mathbf{x}) d \mathbf{x}-\int_{\Omega} p_{h}\left(\mathbf{x}, t_{m}^{p}\right) \nabla \cdot \mathbf{v}_{h}(\mathbf{x}) d \mathbf{x} \\
=\int_{\Omega} \rho g \nabla d(\mathbf{x}) \cdot \mathbf{v}_{h}(\mathbf{x}) d \mathbf{x} & \forall \mathbf{v}_{h}(\mathbf{x}) \in V^{k}, \\
\int_{\Omega} w_{h}(\mathbf{x}) \nabla \cdot \mathbf{u}_{h}\left(\mathbf{x}, t_{m}^{p}\right) d \mathbf{x}=\int_{\Omega} q\left(\mathbf{x}, t_{m}^{p}\right) w_{h}(\mathbf{x}) d \mathbf{x} & \forall w_{h}(\mathbf{x}) \in S_{p}^{k} .
\end{array}
$$

3.3. An ELLAM-MFEM time-stepping procedure. The velocity field usually changes less rapidly than the concentration. Moreover, at each time step the MFEM system (3.12) is more expensive to solve than the ELLAM scheme for the transport PDE (2.2). Therefore, we allow a larger time step for the pressure than that for the concentration [9]. It is often computationally convenient to define the time partition (3.1) for the concentration by subdividing the time partition (3.11) for the pressure. Namely, there exist $0=: N_{0}<N_{1}<\cdots<N_{m}<\cdots<N_{M-1}<N_{M}:=N$ such that $t_{N_{m}}^{c}=t_{m}^{p}$ for $m=1,2, \ldots, M$. For $n=N_{m-1}+1, N_{m-1}+2, \ldots, N_{m}$, the concentration time step $t_{n}^{c}$ relates to the pressure time steps by $t_{m-1}^{p}<t_{n}^{c} \leq t_{m}^{p}$. In the ELLAM scheme we define a velocity approximation $\mathbf{u}_{h}^{\mathrm{E}}\left(\mathbf{x}, t_{n}^{c}\right)$ by an extrapolation of $\mathbf{u}_{h}\left(\mathbf{x}, t_{m-1}^{p}\right)$ and earlier values [9]

$$
\mathbf{u}_{h}^{\mathrm{E}}\left(\mathbf{x}, t_{n}^{c}\right):=\left\{\begin{array}{c}
\left(1+\frac{t_{n}^{c}-t_{m-1}^{p}}{\Delta t_{m-1}^{p}}\right) \mathbf{u}_{h}\left(\mathbf{x}, t_{m-1}^{p}\right)-\frac{t_{n}^{c}-t_{m-1}^{p}}{\Delta t_{m-1}^{p}} \mathbf{u}_{h}\left(\mathbf{x}, t_{m-2}^{p}\right), \\
N_{m-1}+1 \leq n \leq N_{m}, \quad 2 \leq m \leq M \\
\mathbf{u}_{h}(\mathbf{x}, 0), \quad 1 \leq n \leq N_{1}, \quad m=1 .
\end{array}\right.
$$

Let $S_{c}^{l} \subset W_{\infty}^{1}(\Omega)$ be an FEM space, which contains the space of continuous piecewise polynomials of degree at most $l$ on a quasi-uniform partition of diameter $h_{c}$. For example, the FEM space $S_{c}^{l}$ could be the space of piecewise polynomials of degree at most $l$ on triangular elements or the space of piecewise polynomials of degree at most $l$ in each coordinate direction on rectangular elements. We often utilize the fact that velocity is smoother than the concentration to use a much larger grid size $h_{p}$ than $h_{c}$ and to further reduce computational cost since (3.12) is more expensive to solve than (3.14).

Let $c_{h}(\mathbf{x}, 0)$ be an approximation to $c_{0}(\mathbf{x})$ (e.g., its $L^{2}$ or Ritz projection, or interpolation). An ELLAM-MFEM time-stepping procedure is formulated as follows: For $m=1, \ldots, M$, solve the MFEM scheme (3.12) at the pressure time step $t_{m-1}^{p}$. For $n=N_{m-1}+1, N_{m-1}+2, \ldots, N_{m}$, solve the following ELLAM scheme at each 
concentration time step $t_{n}^{c}$ : Find $c_{h}\left(\mathbf{x}, t_{n}^{c}\right) \in S_{c}^{l}$ such that for all $z_{h}\left(\mathbf{x}, t_{n}^{c}\right) \in S_{c}^{l}$

$$
\begin{aligned}
& \int_{\Omega} \phi(\mathbf{x}) c_{h}\left(\mathbf{x}, t_{n}^{c}\right) z_{h}\left(\mathbf{x}, t_{n}^{c}\right) d \mathbf{x} \\
& \quad+\Delta t_{n}^{c} \int_{\Omega} \nabla z_{h}\left(\mathbf{x}, t_{n}^{c}\right) \cdot \mathbf{D}\left(\mathbf{x}, \mathbf{u}_{h}^{E}\left(\mathbf{x}, t_{n}^{c}\right)\right) \nabla c_{h}\left(\mathbf{x}, t_{n}^{c}\right) d \mathbf{x} \\
& =\int_{\Omega} \phi(\mathbf{x}) c_{h}\left(\mathbf{x}, t_{n-1}^{c}\right) z_{h}\left(\mathbf{x}, t_{n-1}^{c,+}\right) d \mathbf{x}+\Delta t_{n}^{c} \int_{\Omega} \bar{c}_{h}\left(\mathbf{x}, t_{n}^{c}\right) q\left(\mathbf{x}, t_{n}^{c}\right) z_{h}\left(\mathbf{x}, t_{n}^{c}\right) d \mathbf{x} .
\end{aligned}
$$

All of the integrals in (3.14), except for the first on the right-hand side, are in Eulerian coordinates and can be evaluated as in FEMs. The first term on the righthand side of (3.14) is in Lagrangian coordinates and requires extra attention. By definition, the test functions $z$ in the ELLAM reference equation (3.9) are constant along the characteristics $\mathbf{r}$ defined by the initial-value problem (3.4). Set

$$
\tilde{\mathbf{x}}:=\mathbf{r}\left(t_{n}^{c} ; \mathbf{x}, t_{n-1}^{c}\right)=\mathbf{x}+\int_{t_{n-1}^{c}}^{t_{n}^{c}} \frac{\mathbf{u}\left(\mathbf{r}\left(\theta ; \mathbf{x}, t_{n-1}^{c}\right), \theta\right)}{\phi\left(\mathbf{r}\left(\theta ; \mathbf{x}, t_{n-1}^{c}\right)\right)} d \theta ;
$$

the test function $z\left(\mathbf{x}, t_{n-1}^{c}\right)$ in (3.9) is determined by

$$
z\left(\mathbf{x}, t_{n-1}^{c,+}\right)=z\left(\tilde{\mathbf{x}}, t_{n}^{c}\right)
$$

Unlike in linear advection-diffusion PDEs $[6,26]$, the velocity $\mathbf{u}$ is unknown. In (3.14) we compute an approximate characteristic $\mathbf{r}_{h}\left(t_{n}^{c} ; \mathbf{x}, t_{n-1}^{c}\right)$ by solving a numerical analogue of problem (3.4)

$$
\frac{d \mathbf{r}_{h}}{d \theta}=\frac{\mathbf{u}_{h}^{\mathrm{E}}\left(\mathbf{r}_{h}, \theta\right)}{\phi\left(\mathbf{r}_{h}\right)} \quad \text { and }\left.\quad \mathbf{r}_{h}(\theta ; \overline{\mathbf{x}}, \bar{t})\right|_{\theta=\bar{t}}=\overline{\mathbf{x}} .
$$

For clarity of exposition, we assume an Euler tracking and set

$$
\begin{aligned}
\tilde{\mathbf{x}}_{h} & :=\mathbf{r}_{h}\left(t_{n}^{c} ; \mathbf{x}, t_{n-1}^{c}\right)=\mathbf{x}+\int_{t_{n-1}^{c}}^{t_{n}^{c}} \frac{\mathbf{u}_{h}^{\mathrm{E}}\left(\mathbf{r}_{h}\left(\theta ; \mathbf{x}, t_{n-1}^{c}\right), \theta\right)}{\phi\left(\mathbf{r}_{h}\left(\theta ; \mathbf{x}, t_{n-1}^{c}\right)\right)} d \theta \\
& =\mathbf{x}+\frac{\mathbf{u}_{h}^{\mathrm{E}}\left(\mathbf{x}, t_{n-1}^{c}\right)}{\phi(\mathbf{x})} \Delta t_{n}^{c} .
\end{aligned}
$$

The test functions $z_{h}\left(\mathbf{x}, t_{n-1}^{c}\right)$ in the ELLAM scheme (3.14) are evaluated by

$$
z_{h}\left(\mathbf{x}, t_{n-1}^{c,+}\right)=z_{h}\left(\tilde{\mathbf{x}}_{h}, t_{n}^{c}\right) .
$$

This further complicates the analysis for the ELLAM-MFEM procedure.

4. Preliminaries. This section cites well-established estimates to be used in the proof of the main theorem in section 5 .

The finite element space $S_{c}^{l}$ has the approximation and inverse properties [7] for $1 \leq m \leq l+1,1 \leq p, q \leq \infty$

$$
\inf _{z_{h} \in S_{c}^{l}}\left(\left\|z-z_{h}\right\|_{L^{q}}+h_{c}\left\|z-z_{h}\right\|_{W_{q}^{1}}\right) \leq A_{1} h_{c}^{m+1+\left(\frac{2}{q}-\frac{2}{p}\right)}\|z\|_{W_{p}^{m}} \forall z \in W_{p}^{m}(\Omega)
$$

and

$$
\begin{array}{ll}
\left\|z_{h}\right\|_{H^{1}} \leq K_{1} h_{c}^{-1}\left\|z_{h}\right\|_{L^{2}}, & \left\|z_{h}\right\|_{L^{\infty}} \leq K_{1}\left|\ln h_{c}\right|^{1 / 2}\left\|z_{h}\right\|_{H^{1}}, \\
\left\|z_{h}\right\|_{W_{q}^{m}} \leq K_{1} h_{c}^{-\left(1-\frac{2}{q}\right)}\left\|z_{h}\right\|_{H^{m}} & \forall z_{h} \in S_{c}^{l}, m=0,1 .
\end{array}
$$

Copyright $@$ by SIAM. Unauthorized reproduction of this article is prohibited. 
The MFEM spaces $\left(V^{k}, S_{p}^{k}\right)$ have the approximation and inverse properties $[4,5$, $7,19]$ for $2 \leq q \leq+\infty$ and $1 \leq m \leq k+1$

$$
\begin{array}{ll}
\inf _{\mathbf{v} h \in V^{k}}\left\|\mathbf{v}-\mathbf{v}_{h}\right\|_{L^{q}} \leq A_{2} h_{p}^{m-\left(1-\frac{2}{q}\right)}\|\mathbf{v}\|_{H^{m}} & \forall \mathbf{v} \in H^{m}, \\
\inf _{\mathbf{v}_{h} \in V^{k}}\left\|\mathbf{v}-\mathbf{v}_{h}\right\|_{H(\text { div })} \leq A_{2} h_{p}^{m}\|\mathbf{v}\|_{H^{m}(\text { div })} & \forall \mathbf{v} \in H^{m}(\operatorname{div}), \\
\inf _{g_{h} \in S_{p}^{k}}\left\|g-g_{h}\right\|_{L^{2}} \leq A_{2} h_{p}^{m}\|g\|_{H^{m}} & \forall g \in H^{m}
\end{array}
$$

and

$$
\begin{array}{ll}
\left\|\mathbf{v}_{h}\right\|_{L^{q}} \leq K_{2} h_{p}^{-\left(1-\frac{2}{q}\right)}\left\|\mathbf{v}_{h}\right\|_{L^{2}} & \forall \mathbf{v}_{h} \in V^{k} \\
\left\|\mathbf{v}_{h}\right\|_{W_{q}^{1}} \leq K_{2} h_{p}^{-1}\left\|\mathbf{v}_{h}\right\|_{L^{q}} \quad \forall \mathbf{v}_{h} \in V^{k} .
\end{array}
$$

It is understood in (4.4) that

$$
\left\|\mathbf{v}_{h}\right\|_{W_{q}^{1}}:= \begin{cases}\left(\sum_{\Omega_{e}^{p} \subset \Omega}\left\|\mathbf{v}_{h}\right\|_{W_{q}^{1}\left(\Omega_{e}^{p}\right)}^{q}\right)^{1 / q} & \text { for } 2 \leq q<+\infty \\ \max _{\forall \Omega_{e}^{p} \subset \Omega}\left\|\mathbf{v}_{h}\right\|_{W_{q}^{1}\left(\Omega_{e}^{p}\right)} & \text { for } q=+\infty\end{cases}
$$

where $\Omega_{e}^{p} \subset \Omega$ are all of the elements of the pressure mesh.

Let $\Pi c(\mathbf{x}, t) \in S_{c}^{l}, t \in[0, T]$, be the Ritz projection of $c(\mathbf{x}, t)$ defined by $[23,33]$

$$
\begin{aligned}
& \int_{\Omega} \chi(\mathbf{x})(\Pi c(\mathbf{x}, t)-c(\mathbf{x}, t)) d \mathbf{x} \\
& \quad+\int_{\Omega} \nabla \chi(\mathbf{x}) \cdot \mathbf{D}(\mathbf{x}, \mathbf{u}(\mathbf{x}, t)) \nabla(\Pi c(\mathbf{x}, t)-c(\mathbf{x}, t)) d \mathbf{x}=0 \quad \forall \chi \in S_{c}^{l} .
\end{aligned}
$$

The following estimates hold $[7,19,23,33]$ for $2 \leq q \leq+\infty, 1 \leq m \leq l+1$ :

$$
\begin{aligned}
\|\Pi c-c\|_{L^{\infty}\left(L^{q}\right)}+h_{c}\|\Pi c-c\|_{L^{\infty}\left(W_{q}^{1}\right)} & \leq A_{1} h_{c}^{m}\|c\|_{L^{\infty}\left(W_{q}^{m}\right)}, \\
\|\Pi c-c\|_{H^{1}\left(L^{q}\right)} & \leq A_{1} h_{c}^{m}\|c\|_{H^{1}\left(W_{q}^{m}\right)} .
\end{aligned}
$$

Here the constant $A_{1}$ is independent of $c$ and $h_{c}$.

Let $\mathrm{I} c(\mathbf{x}, t) \in S_{c}^{l}, t \in[0, T]$, be the interpolation of $c(\mathbf{x}, t)$. We use the estimates (4.1) with $p=2$ and $q=+\infty$, (4.2) with $q=+\infty$, and (4.6) with $q=2$ to conclude that for $c \in L^{\infty}\left(W_{\infty}^{1} \cap H^{2}\right)$

$$
\begin{aligned}
\|\Pi c\|_{L^{\infty}\left(W_{\infty}^{1}\right)} \leq & \|\Pi c-\mathrm{I} c\|_{L^{\infty}\left(W_{\infty}^{1}\right)}+\|\mathrm{I} c-c\|_{L^{\infty}\left(W_{\infty}^{1}\right)}+\|c\|_{L^{\infty}\left(W_{\infty}^{1}\right)} \\
\leq & K_{1} h_{c}^{-1}\|\Pi c-\mathrm{I} c\|_{L^{\infty}\left(H^{1}\right)}+\left(A_{1}+1\right)\|c\|_{L^{\infty}\left(W_{\infty}^{1}\right)} \\
\leq & K_{1} h_{c}^{-1}\left(\|\Pi c-c\|_{L^{\infty}\left(H^{1}\right)}+\|\mathrm{I} c-c\|_{L^{\infty}\left(H^{1}\right)}\right) \\
& +\left(A_{1}+1\right)\|c\|_{L^{\infty}\left(W_{\infty}^{1}\right)} \\
\leq & 2 A_{1} K_{1}\|c\|_{L^{\infty}\left(H^{2}\right)}+\left(A_{1}+1\right)\|c\|_{L^{\infty}\left(W_{\infty}^{1}\right)}=: K_{3} .
\end{aligned}
$$

Copyright $@$ by SIAM. Unauthorized reproduction of this article is prohibited. 
We similarly define a mapping $(\Pi \mathbf{u}, \Pi p)$ from $H($ div $) \times L_{0}^{2}$ to $V^{k} \times S_{p}^{k}$ by

$$
\begin{aligned}
\int_{\Omega} \mu(c(\mathbf{x}, t)) \mathbf{K}^{-1}(\mathbf{x})(\Pi \mathbf{u}(\mathbf{x}, t)-\mathbf{u}(\mathbf{x}, t)) \cdot \mathbf{v}_{h}(\mathbf{x}) d \mathbf{x} & \\
-\int_{\Omega}(\Pi p(\mathbf{x}, t)-p(\mathbf{x}, t)) \nabla \cdot \mathbf{v}_{h}(\mathbf{x}) d \mathbf{x}=0 & \forall \mathbf{v}_{h} \in V^{k}, \\
\int_{\Omega} w_{h}(\mathbf{x}) \nabla \cdot(\Pi \mathbf{u}(\mathbf{x}, t)-\mathbf{u}(\mathbf{x}, t)) d \mathbf{x}=0 & \forall w_{h} \in S_{p}^{k} .
\end{aligned}
$$

The following estimates hold, e.g., for Raviart-Thomas spaces [4, 8, 9]:

$$
\begin{aligned}
& \|\Pi \mathbf{u}-\mathbf{u}\|_{L^{\infty}(H(\mathrm{div}))}+\|\Pi p-p\|_{L^{\infty}\left(L^{2}\right)} \\
& \quad \leq A\left(\inf _{\mathbf{v}_{h} \in V^{k}}\left\|\mathbf{u}-\mathbf{v}_{h}\right\|_{L^{\infty}(H(\mathrm{div}))}+\inf _{g_{h} \in S_{p}^{k}}\left\|p-g_{h}\right\|_{L^{\infty}\left(L^{2}\right)}\right) \\
& \quad \leq A_{2} h_{p}^{k+1}\left(\|\mathbf{u}\|_{L^{\infty}\left(H^{k+1}(\mathrm{div})\right)}+\|p\|_{L^{\infty}\left(H^{k+1}\right)}\right) .
\end{aligned}
$$

Here $A_{2}$ is independent of $h_{p}, u, p$, and $c$.

We let I be an interpolation operator from $H(\operatorname{div}) \times L_{0}^{2}$ to $V^{k} \times S_{p}^{k}$ for $t \in[0, T]$. We use the estimates (4.4) and (4.9) to conclude that

$$
\begin{aligned}
\|\Pi \mathbf{u}\|_{L^{\infty}\left(L^{\infty}\right)} \leq & \|\Pi \mathbf{u}-\mathrm{I} \mathbf{u}\|_{L^{\infty}\left(L^{\infty}\right)}+\|\mathbf{I} \mathbf{u}-\mathbf{u}\|_{L^{\infty}\left(L^{\infty}\right)}+\|\mathbf{u}\|_{L^{\infty}\left(L^{\infty}\right)} \\
\leq & K_{2} h_{p}^{-1}\|\Pi \mathbf{u}-\mathrm{I} \mathbf{u}\|_{L^{\infty}\left(L^{2}\right)}+\left(A_{2}+1\right)\|\mathbf{u}\|_{L^{\infty}\left(L^{\infty}\right)} \\
\leq & K_{2} h_{p}^{-1}\left(\|\Pi \mathbf{u}-\mathbf{u}\|_{L^{\infty}\left(L^{2}\right)}+\|\mathbf{I} \mathbf{u}-\mathbf{u}\|_{L^{\infty}\left(L^{2}\right)}\right) \\
& +\left(A_{2}+1\right)\|\mathbf{u}\|_{L^{\infty}\left(L^{\infty}\right)} \\
\leq & 2 A_{2} K_{2}\left(\|\mathbf{u}\|_{L^{\infty}\left(H^{1}(\operatorname{div})\right)}+\|p\|_{L^{\infty}\left(H^{1}\right)}\right) \\
& +\left(A_{2}+1\right)\|\mathbf{u}\|_{L^{\infty}\left(L^{\infty}\right)}=: K_{4} .
\end{aligned}
$$

For the analysis in section 5 we introduce an extrapolation of the exact velocity $\mathbf{u}$

$$
\mathbf{u}^{\mathrm{E}}\left(\mathbf{x}, t_{n}^{c}\right):=\left\{\begin{array}{c}
\left(1+\frac{t_{n}^{c}-t_{m-1}^{p}}{\Delta t_{m-1}^{p}}\right) \mathbf{u}\left(\mathbf{x}, t_{m-1}^{p}\right)-\frac{t_{n}^{c}-t_{m-1}^{p}}{\Delta t_{m-1}^{p}} \mathbf{u}\left(\mathbf{x}, t_{m-2}^{p}\right), \\
N_{m-1}+1 \leq n \leq N_{m}, \quad 2 \leq m \leq M \\
\mathbf{u}(\mathbf{x}, 0), \quad 1 \leq n \leq N_{1}, \quad m=1 .
\end{array}\right.
$$

Then we routinely see that for $2 \leq q \leq+\infty$

$$
\begin{aligned}
& \left\|\mathbf{u}^{E}(\mathbf{x}, t)-\mathbf{u}(\mathbf{x}, t)\right\|_{L^{q}} \\
& \quad \leq \begin{cases}A_{3}\left(\Delta t_{p}\right)^{\frac{3}{2}}\|\mathbf{u}\|_{H^{2}\left(t_{m-2}^{p}, t_{m}^{p} ; L^{q}\right)} & \forall t \in\left[t_{m-1}^{p}, t_{m}^{p}\right], m \geq 2, \\
A_{3} \Delta t_{p}^{1}\|\mathbf{u}\|_{W_{1}^{\infty}\left(t_{0}^{p}, t_{1}^{p} ; L^{q}\right)} & \forall t \in\left[t_{0}^{p}, t_{1}^{p}\right], \quad m=1 .\end{cases}
\end{aligned}
$$

5. An optimal-order error estimate. In this section we prove an optimalorder error estimate for the ELLAM-MFEM time-stepping procedure with any order of approximating polynomials $(k \geq 0, l \geq 1)$.

5.1. On the main theorem and its proof. Note that the test functions in the ELLAM formulation are defined by (3.3), which can be evaluated by using (3.19). However, the velocity $\mathbf{u}$ is unknown. This is in contrast to the ELLAM schemes for 
linear transport PDEs in which the true velocity is known and is used to evaluate characteristics $[6,21,26]$. In the ELLAM-MFEM time-stepping procedure in section 3 , we have to use the numerical velocity $\mathbf{u}_{h}^{\mathrm{E}}$ to evaluate the approximate characteristics $\mathbf{r}_{h}$ by solving problem (3.17). These issues further complicate the analysis [21, 25].

THEOREM 5.1. Suppose that the solution $(c, p, \mathbf{u})$ of problem (2.1)-(2.3) satisfies $c \in L^{\infty}\left(W_{2+\gamma}^{l+1}\right) \cap L^{\infty}\left(W_{\infty}^{1}\right) \cap H^{1}\left(H^{l+1}\right)$ for some $\gamma>0, p \in L^{\infty}\left(H^{k+1}\right)$, and $\mathbf{u} \in$ $L^{\infty}\left(H^{k+1}(\operatorname{div}) \cap W_{\infty}^{1}\right) \cap W_{\infty}^{1}\left(L^{\infty}\right) \cap H^{2}\left(L^{2}\right)$. Let $\left(c_{h}\left(\mathbf{x}, t_{n}^{c}\right), p_{h}\left(\mathbf{x}, t_{m}^{p}\right), \mathbf{u}_{h}\left(\mathbf{x}, t_{m}^{p}\right)\right)$ be the solution of the ELLAM-MFEM time-stepping procedure (3.12) and (3.14) with $l \geq 1$ and $k \geq 0$. Assume that the discretization parameters obey the relations

$$
\Delta t_{c}=O\left(h_{p}\right), \quad h_{c}^{l+1}=o\left(h_{p}\right), \quad \Delta t_{p}^{1}=O\left(h_{p}^{2 / 3}\right), \quad \Delta t_{p}=O\left(h_{p}^{1 / 2}\right) .
$$

There exist positive constants $h_{c}^{*}, h_{p}^{*}, \Delta t_{c}^{*}, \Delta t_{p}^{*}$, and $Q^{*}$ such that the following optimal-order error estimate holds for $0<h_{c} \leq h_{c}^{*}, 0<h_{p} \leq h_{p}^{*}, 0<\Delta t_{c} \leq \Delta t_{c}^{*}$, and $0<\Delta t_{p} \leq \Delta t_{p}^{*}$ :

$$
\begin{aligned}
\| c_{h}- & c\left\|_{\hat{L}_{c}^{\infty}\left(L^{2}\right)}+|\mathbf{D}|_{\text {min }}^{1 / 2}\right\| c_{h}-c \|_{\hat{L}_{c}^{2}\left(H^{1}\right)} \\
& +\left\|\mathbf{u}_{h}-\mathbf{u}\right\|_{\hat{L}_{p}^{\infty}(H(\mathrm{div}))}+\left\|p_{h}-p\right\|_{\hat{L}_{p}^{\infty}\left(L^{2}\right)} \\
\leq & Q^{*} \Delta t_{c}\left(\|\bar{c}\|_{L^{2}\left(L^{2}\right)}+\|c\|_{L^{2}\left(H^{1}\right)}+\left\|\frac{d \bar{c}}{d t}\right\|_{L^{2}\left(L^{2}\right)}+\left\|\frac{d c}{d t}\right\|_{L^{2}\left(H^{1}\right)}\right) \\
& +Q^{*}\left(\left(\Delta t_{p}^{1}\right)^{3 / 2}+\left(\Delta t_{p}\right)^{2}\right)\|\mathbf{u}\|_{H^{2}\left(L^{2}\right)}+Q^{*} h_{c}^{l+1}\left(\|c\|_{L^{\infty}\left(W_{2+\gamma}^{l+1}\right)}\right. \\
& \left.+\|c\|_{H^{1}\left(H^{l+1}\right)}\right)+Q^{*} h_{p}^{k+1}\left(\|\mathbf{u}\|_{L^{\infty}\left(H^{k+1}(\operatorname{div})\right)}+\|p\|_{L^{\infty}\left(H^{k+1}\right)}\right) .
\end{aligned}
$$

Here $\frac{d}{d t}$ refers to the material derivative along the characteristics defined in (3.4). The constant $Q^{*}=Q^{*}\left(h_{c}^{*}, h_{p}^{*}, \Delta t_{c}^{*}, \Delta t_{p}^{*}, T\right)$, but $Q^{*}$ is independent of the discretization parameters $h_{c}, h_{p}, \Delta t_{c}$, or $\Delta t_{p}$.

To prove the theorem, we use (3.10), (3.12), and (4.8) to derive a relation

$$
\begin{aligned}
& \int_{\Omega} \mu(\left.c_{h}\left(\mathbf{x}, t_{m}^{p}\right)\right) \mathbf{K}^{-1}(\mathbf{x})\left(\mathbf{u}_{h}\left(\mathbf{x}, t_{m}^{p}\right)-\Pi \mathbf{u}\left(\mathbf{x}, t_{m}^{p}\right)\right) \cdot \mathbf{v}_{h}(\mathbf{x}) d \mathbf{x} \\
&-\int_{\Omega}\left(p_{h}\left(\mathbf{x}, t_{m}^{p}\right)-\Pi p\left(\mathbf{x}, t_{m}^{p}\right)\right) \nabla \cdot \mathbf{v}_{h}(\mathbf{x}) d \mathbf{x} \\
&= \int_{\Omega}\left(\mu\left(c\left(\mathbf{x}, t_{m}^{p}\right)\right)-\mu\left(c_{h}\left(\mathbf{x}, t_{m}^{p}\right)\right)\right) \mathbf{K}^{-1}(\mathbf{x}) \Pi \mathbf{u}\left(\mathbf{x}, t_{m}^{p}\right) \cdot \mathbf{v}_{h}(\mathbf{x}) d \mathbf{x}, \\
& \int_{\Omega} w_{h}(\mathbf{x}) \nabla \cdot\left(\mathbf{u}_{h}\left(\mathbf{x}, t_{m}^{p}\right)-\Pi \mathbf{u}\left(\mathbf{x}, t_{m}^{p}\right)\right) d \mathbf{x}=0 \quad \forall\left(\mathbf{v}_{h}, w_{h}\right) \in V^{k} \times S_{p}^{k} .
\end{aligned}
$$

Combining this equation with (4.10) yields an estimate $[4,5,8]$

$$
\begin{gathered}
\left\|\mathbf{u}_{h}\left(\mathbf{x}, t_{m}^{p}\right)-\Pi \mathbf{u}\left(\mathbf{x}, t_{m}^{p}\right)\right\|_{H(\mathrm{div})}+\left\|p_{h}\left(\mathbf{x}, t_{m}^{p}\right)-\Pi p\left(\mathbf{x}, t_{m}^{p}\right)\right\| \\
\leq Q\left(1+\left\|\Pi \mathbf{u}\left(\mathbf{x}, t_{m}^{p}\right)\right\|_{L^{\infty}}\right)\left\|c_{h}\left(\mathbf{x}, t_{m}^{p}\right)-c\left(\mathbf{x}, t_{m}^{p}\right)\right\| \\
\leq Q_{1}\left\|c_{h}\left(\mathbf{x}, t_{m}^{p}\right)-c\left(\mathbf{x}, t_{m}^{p}\right)\right\|, \quad 0 \leq m \leq M .
\end{gathered}
$$

For convenience, we have dropped the subscript $L^{2}$.

Copyright $@$ by SIAM. Unauthorized reproduction of this article is prohibited. 
The estimates (4.9) and (5.3) show that the bound on $\left\|\mathbf{u}_{h}-\mathbf{u}\right\|_{\hat{L}_{p}^{\infty}(H(\mathrm{div}))}+\| p_{h}-$ $p \|_{\hat{L}_{p}^{\infty}\left(L^{2}\right)}$ in (5.2) is a consequence of the bound on $\left\|c_{h}-c\right\|_{\hat{L}_{c}^{\infty}\left(L^{2}\right)}$.

5.2. Proof of the key estimate. To bound $\left\|c_{h}-c\right\|_{\hat{L}_{c}^{\infty}\left(L^{2}\right)}$, we set $\xi\left(\mathbf{x}, t_{n}^{c}\right):=$ $c_{h}\left(\mathbf{x}, t_{n}^{c}\right)-\Pi c\left(\mathbf{x}, t_{n}^{c}\right)$ and $\eta\left(\mathbf{x}, t_{n}^{c}\right):=\Pi c\left(\mathbf{x}, t_{n}^{c}\right)-c\left(\mathbf{x}, t_{n}^{c}\right)$. Note that $c_{h}-c=\xi+\eta$ and that the estimate for $\eta$ is known from (4.6). The key to prove the theorem is to derive an estimate of the form (5.2) for $\xi$.

Proof. We subtract (3.14) from (3.9) for $n=N_{m-1}+1, \ldots, N_{m}$ to generate an error equation for any $z_{h}\left(\mathbf{x}, t_{n}^{c}\right) \in S_{c}^{l}$

$$
\begin{aligned}
& \int_{\Omega} \phi(\mathbf{x})\left(c_{h}\left(\mathbf{x}, t_{n}^{c}\right)-c\left(\mathbf{x}, t_{n}^{c}\right)\right) z_{h}\left(\mathbf{x}, t_{n}^{c}\right) d \mathbf{x} \\
& \quad+\Delta t_{n}^{c} \int_{\Omega} \nabla z_{h}\left(\mathbf{x}, t_{n}^{c}\right) \cdot\left(\mathbf{D}\left(\mathbf{x}, \mathbf{u}_{h}^{E}\left(\mathbf{x}, t_{n}^{c}\right)\right) \nabla c_{h}\left(\mathbf{x}, t_{n}^{c}\right)-\mathbf{D}\left(\mathbf{x}, \mathbf{u}\left(\mathbf{x}, t_{n}^{c}\right)\right) \nabla c\left(\mathbf{x}, t_{n}^{c}\right)\right) d \mathbf{x} \\
& =\int_{\Omega} \phi(\mathbf{x})\left(c_{h}\left(\mathbf{x}, t_{n-1}^{c}\right)-c\left(\mathbf{x}, t_{n-1}^{c}\right)\right) z_{h}\left(\mathbf{x}, t_{n-1}^{c,+}\right) d \mathbf{x} \\
& \quad+\Delta t_{n}^{c} \int_{\Omega}\left(\bar{c}_{h}\left(\mathbf{x}, t_{n}^{c}\right)-\bar{c}\left(\mathbf{x}, t_{n}^{c}\right)\right) q\left(\mathbf{x}, t_{n}^{c}\right) z_{h}\left(\mathbf{x}, t_{n}^{c}\right) d \mathbf{x} \\
& \quad+E_{\mathbf{D}}\left(c, z_{h}\right)-E_{q}\left(\bar{c}, z_{h}\right) .
\end{aligned}
$$

In the second term on the right-hand side, $\bar{c}_{h}:=\bar{c}$ is specified at sources so the difference $\bar{c}_{h}-\bar{c}$ vanishes. At sinks $q=q^{-}$is specified but $\bar{c}_{h}=c_{h}$ and $\bar{c}=c$. We rewrite the preceding equation with $z_{h}=\xi$ as

$$
\begin{aligned}
& \int_{\Omega} \phi(\mathbf{x}) \xi^{2}\left(\mathbf{x}, t_{n}^{c}\right) d \mathbf{x}+\Delta t_{n}^{c} \int_{\Omega} \nabla \xi\left(\mathbf{x}, t_{n}^{c}\right) \cdot \mathbf{D}\left(\mathbf{x}, \mathbf{u}_{h}^{E}\left(\mathbf{x}, t_{n}^{c}\right)\right) \nabla \xi\left(\mathbf{x}, t_{n}^{c}\right) d \mathbf{x} \\
& =\int_{\Omega} \phi(\mathbf{x}) \xi\left(\mathbf{x}, t_{n-1}^{c}\right) \xi\left(\mathbf{x}, t_{n-1}^{c,+}\right) d \mathbf{x}+\int_{\Omega} \phi(\mathbf{x}) \eta\left(\mathbf{x}, t_{n-1}^{c}\right) \xi\left(\mathbf{x}, t_{n-1}^{c,+}\right) d \mathbf{x} \\
& \quad+\Delta t_{n}^{c} \int_{\Omega} q^{-}\left(\mathbf{x}, t_{n}^{c}\right) \xi^{2}\left(\mathbf{x}, t_{n}^{c}\right) d \mathbf{x}+\Delta t_{n}^{c} \int_{\Omega} q^{-}\left(\mathbf{x}, t_{n}^{c}\right) \eta\left(\mathbf{x}, t_{n}^{c}\right) \xi\left(\mathbf{x}, t_{n}^{c}\right) d \mathbf{x} \\
& \quad-\Delta t_{n}^{c} \int_{\Omega} \nabla \xi\left(\mathbf{x}, t_{n}^{c}\right) \cdot\left(\mathbf{D}\left(\mathbf{x}, \mathbf{u}_{h}^{E}\left(\mathbf{x}, t_{n}^{c}\right)\right)-\mathbf{D}\left(\mathbf{x}, \mathbf{u}\left(\mathbf{x}, t_{n}^{c}\right)\right)\right) \nabla \Pi c\left(\mathbf{x}, t_{n}^{c}\right) d \mathbf{x} \\
& \quad-\Delta t_{n}^{c} \int_{\Omega} \nabla \xi\left(\mathbf{x}, t_{n}^{c}\right) \cdot \mathbf{D}\left(\mathbf{x}, \mathbf{u}\left(\mathbf{x}, t_{n}^{c}\right)\right) \nabla \eta\left(\mathbf{x}, t_{n}^{c}\right) d \mathbf{x} \\
& \quad-\int_{\Omega} \phi(\mathbf{x}) \eta\left(\mathbf{x}, t_{n}^{c}\right) \xi\left(\mathbf{x}, t_{n}^{c}\right) d \mathbf{x}+E_{\mathbf{D}}(c, \xi)-E_{q}(\bar{c}, \xi) .
\end{aligned}
$$

We bound the third and fourth terms on the right-hand side of (5.4) by

$$
\begin{aligned}
& \left|\Delta t_{n}^{c} \int_{\Omega} q^{-}\left(\mathbf{x}, t_{n}^{c}\right) \xi^{2}\left(\mathbf{x}, t_{n}^{c}\right) d \mathbf{x}+\Delta t_{n}^{c} \int_{\Omega} q^{-}\left(\mathbf{x}, t_{n}^{c}\right) \eta\left(\mathbf{x}, t_{n}^{c}\right) \xi\left(\mathbf{x}, t_{n}^{c}\right) d \mathbf{x}\right| \\
& \quad \leq Q \Delta t_{n}^{c}\left\|\xi\left(\mathbf{x}, t_{n}^{c}\right)\right\|^{2}+\Delta t_{n}^{c}\left\|\eta\left(\mathbf{x}, t_{n}^{c}\right)\right\|^{2} \\
& \quad \leq Q \Delta t_{n}^{c}\left\|\xi\left(\mathbf{x}, t_{n}^{c}\right)\right\|^{2}+A_{1}^{2} \Delta t_{n}^{c} h_{c}^{2 l+2}\|c\|_{L^{\infty}\left(H^{l+1}\right)}^{2}
\end{aligned}
$$

Copyright $\odot$ by SIAM. Unauthorized reproduction of this article is prohibited. 
We use (4.5) and (4.6) to bound the sixth term on the right-hand side of (5.4)

$$
\begin{aligned}
& \left|\Delta t_{n}^{c} \int_{\Omega} \nabla \xi\left(\mathbf{x}, t_{n}^{c}\right) \cdot \mathbf{D}\left(\mathbf{x}, \mathbf{u}\left(\mathbf{x}, t_{n}^{c}\right)\right) \nabla \eta\left(\mathbf{x}, t_{n}^{c}\right) d \mathbf{x}\right| \\
& \quad=\left|\Delta t_{n}^{c} \int_{\Omega} \xi\left(\mathbf{x}, t_{n}^{c}\right) \eta\left(\mathbf{x}, t_{n}^{c}\right) d \mathbf{x}\right| \\
& \quad \leq Q \Delta t_{n}^{c}\left\|\xi\left(\mathbf{x}, t_{n}^{c}\right)\right\|^{2}+\Delta t_{n}^{c}\left\|\eta\left(\mathbf{x}, t_{n}^{c}\right)\right\|^{2} \\
& \quad \leq Q \Delta t_{n}^{c}\left\|\xi\left(\mathbf{x}, t_{n}^{c}\right)\right\|^{2}+A_{1}^{2} \Delta t_{n}^{c} h_{c}^{2 l+2}\|c\|_{L^{\infty}\left(H^{l+1}\right)}^{2} .
\end{aligned}
$$

The fifth term on the right-hand side of (5.4) is bounded in Lemma 6.3

$$
\begin{aligned}
& \left|\Delta t_{n}^{c} \int_{\Omega} \nabla \xi\left(\mathbf{x}, t_{n}^{c}\right) \cdot\left(\mathbf{D}\left(\mathbf{x}, \mathbf{u}_{h}^{E}\left(\mathbf{x}, t_{n}^{c}\right)\right)-\mathbf{D}\left(\mathbf{x}, \mathbf{u}\left(\mathbf{x}, t_{n}^{c}\right)\right)\right) \nabla \Pi c\left(\mathbf{x}, t_{n}^{c}\right) d \mathbf{x}\right| \\
& \leq \varepsilon \Delta t_{n}^{c}\left\|\nabla \xi\left(\mathbf{x}, t_{n}^{c}\right)\right\|^{2}+Q \Delta t_{n}^{c}\left(\left\|\xi\left(\mathbf{x}, t_{m-1}^{p}\right)\right\|^{2}+\left\|\xi\left(\mathbf{x}, t_{m-2}^{p}\right)\right\|^{2}\right) \\
& +Q \Delta t_{n}^{c}\left(h_{c}^{2 l+2}\|c\|_{L^{\infty}\left(H^{l+1}\right)}^{2}+h_{p}^{2 k+2}\left(\|\mathbf{u}\|_{L^{\infty}\left(H^{k+1}(\mathrm{div})\right)}^{2}+\|p\|_{L^{\infty}\left(H^{k+1}\right)}^{2}\right)\right. \\
& \left.+\delta_{m, 1}\left(\Delta t_{p}^{1}\right)^{2}\|\mathbf{u}\|_{W_{1}^{\infty}\left(0, t_{1}^{p} ; L^{2}\right)}^{2}+\left(1-\delta_{m, 1}\right)\left(\Delta t_{p}\right)^{3}\|\mathbf{u}\|_{H^{2}\left(t_{m-2}^{p}, t_{m}^{p} ; L^{2}\right)}^{2}\right),
\end{aligned}
$$

where $\delta_{i, j}=1$ if $i=j$ or 0 otherwise.

Note that the evaluation of source and sink terms and the diffusion-dispersion term in the ELLAM scheme (3.14) does not involve any characteristic tracking. Hence, (3.6) and (3.8) still hold, leading to the estimate

$$
\begin{aligned}
\left|E_{q}(\bar{c}, \xi)\right|= & \mid \int_{\Omega} \xi\left(\mathbf{x}, t_{n}^{c}\right) \int_{t_{n-1}^{c}}^{t_{n}^{c}}\left\{\int_{\theta}^{t_{n}^{c}} \frac{d}{d t}\left(\bar{c}\left(\mathbf{r}\left(t ; \mathbf{x}, t_{n}^{c}\right), t\right) q\left(\mathbf{r}\left(t ; \mathbf{x}, t_{n}^{c}\right), t\right)\right) d t\right. \\
& \left.+\bar{c}\left(\mathbf{r}\left(\theta ; \mathbf{x}, t_{n}^{c}\right), \theta\right) q\left(\mathbf{r}\left(\theta ; \mathbf{x}, t_{n}^{c}\right), \theta\right)\left[\operatorname{det}\left(\frac{\partial \mathbf{r}\left(\theta ; \mathbf{x}, t_{n}^{c}\right)}{\partial \mathbf{x}}\right)-1\right]\right\} d \theta d \mathbf{x} \mid \\
\leq & Q \Delta t_{n}^{c}\left\|\xi\left(\mathbf{x}, t_{n}^{c}\right)\right\|^{2}+Q\left(\Delta t_{n}^{c}\right)^{2}\left(\|\bar{c}\|_{L^{2}\left(t_{n-1}^{c}, t_{n}^{c} ; L^{2}\right)}^{2}+\left\|\frac{d \bar{c}}{d t}\right\|_{L^{2}\left(t_{n-1}^{c}, t_{n}^{c} ; L^{2}\right)}^{2}\right) .
\end{aligned}
$$

We use the estimates (6.1) and (6.2) in Lemma 6.1 to bound $\left|E_{\mathbf{D}}(c, \xi)\right|$ by

$$
\begin{aligned}
&\left|E_{\mathbf{D}}(c, \xi)\right| \leq \mid \int_{\Omega} \int_{t_{n-1}^{c}}^{t_{n}^{c}}\left(\operatorname{det}\left(\frac{\partial \mathbf{r}\left(\theta ; \mathbf{x}, t_{n}^{c}\right)}{\partial \mathbf{x}}\right)\left(\frac{\partial \mathbf{r}\left(\theta ; \mathbf{x}, t_{n}^{c}\right)}{\partial \mathbf{x}}\right)^{-1}-\mathbf{I}\right) \nabla \xi\left(\mathbf{x}, t_{n}^{c}\right) \cdot\left(\mathbf{D}\left(\mathbf{r}\left(\theta ; \mathbf{x}, t_{n}^{c}\right), \mathbf{u}\left(\mathbf{r}\left(\theta ; \mathbf{x}, t_{n}^{c}\right), \theta\right)\right) \nabla c\left(\mathbf{r}\left(\theta ; \mathbf{x}, t_{n}^{c}\right), \theta\right)\right) d \theta d \mathbf{x} \mid \\
&+\mid \int_{\Omega} \int_{t_{n-1}^{c}}^{t_{n}^{c}} \nabla \xi\left(\mathbf{x}, t_{n}\right) \cdot\left(\mathbf{D}\left(\mathbf{x}, \mathbf{u}\left(\mathbf{x}, t_{n}^{c}\right)\right) \nabla c\left(\mathbf{x}, t_{n}^{c}\right)\right. \\
&\left.\quad-\mathbf{D}\left(\mathbf{r}\left(\theta ; \mathbf{x}, t_{n}^{c}\right), \mathbf{u}\left(\mathbf{r}\left(\theta ; \mathbf{x}, t_{n}^{c}\right), \theta\right)\right) \nabla c\left(\mathbf{r}\left(\theta ; \mathbf{x}, t_{n}^{c}\right), \theta\right)\right) d \theta d \mathbf{x} \mid \\
& \leq \varepsilon \Delta t_{n}^{c}\left\|\nabla \xi\left(\mathbf{x}, t_{n}^{c}\right)\right\|^{2}+Q\left(\Delta t_{n}^{c}\right)^{2}\left(\|c\|_{L^{2}\left(t_{n-1}^{c}, t_{n}^{c} ; H^{1}\right)}^{2}+\left\|\frac{d c}{d t}\right\|_{L^{2}\left(t_{n-1}^{c}, t_{n}^{c} ; H^{1}\right)}^{2}\right) .
\end{aligned}
$$

Copyright (c) by SIAM. Unauthorized reproduction of this article is prohibited. 
The first term on the right-hand side of (5.4) is estimated in Lemma 6.4

$$
\begin{aligned}
& \left|\int_{\Omega} \phi(\mathbf{x}) \xi\left(\mathbf{x}, t_{n-1}^{c}\right) \xi\left(\mathbf{x}, t_{n-1}^{c,+}\right) d \mathbf{x}\right| \\
& \leq \frac{1}{2} \int_{\Omega} \phi(\mathbf{x}) \xi^{2}\left(\mathbf{x}, t_{n-1}^{c}\right) d \mathbf{x}+\frac{1}{2} \int_{\Omega} \phi(\mathbf{x}) \xi^{2}\left(\mathbf{x}, t_{n}^{c}\right) d \mathbf{x}+\varepsilon \Delta t_{n}^{c}\left(\left\|\nabla \xi\left(\mathbf{x}, t_{n}^{c}\right)\right\|^{2}\right. \\
& \left.\quad+\left\|\nabla \xi\left(\mathbf{x}, t_{n-1}^{c}\right)\right\|^{2}\right)+Q \Delta t_{n}^{c}\left(\left\|\xi\left(\mathbf{x}, t_{n}^{c}\right)\right\|^{2}+\left\|\xi\left(\mathbf{x}, t_{n-1}^{c}\right)\right\|^{2}\right) .
\end{aligned}
$$

The second and seventh terms on the right-hand side of (5.4) are estimated in Lemma 6.5

$$
\begin{aligned}
& \left|\int_{\Omega} \phi(\mathbf{x}) \eta\left(\mathbf{x}, t_{n}^{c}\right) \xi\left(\mathbf{x}, t_{n}^{c}\right) d \mathbf{x}-\int_{\Omega} \phi(\mathbf{x}) \eta\left(\mathbf{x}, t_{n-1}^{c}\right) \xi\left(\mathbf{x}, t_{n-1}^{c,+}\right) d \mathbf{x}\right| \\
& \quad \leq \varepsilon \Delta t_{n}^{c}\left\|\nabla \xi\left(\mathbf{x}, t_{n}^{c}\right)\right\|^{2}+Q \Delta t_{n}^{c}\left\|\xi\left(\mathbf{x}, t_{n}^{c}\right)\right\|^{2} \\
& \quad+Q h_{c}^{2 l+2}\|c\|_{H^{1}\left(t_{n-1}^{c}, t_{n}^{c} ; H^{l+1}\right)}^{2}+Q \Delta t_{n}^{c} h_{c}^{2 l+2}\|c\|_{L^{\infty}\left(W_{2+\gamma}^{l+1}\right)}^{2}
\end{aligned}
$$

We incorporate the preceding estimates into (5.4) to get

$$
\begin{aligned}
\int_{\Omega} \phi & (\mathbf{x}) \xi^{2}\left(\mathbf{x}, t_{n}^{c}\right) d \mathbf{x}+|\mathbf{D}|_{\min } \Delta t_{n}^{c}\left\|\nabla \xi\left(\mathbf{x}, t_{n}^{c}\right)\right\|^{2} \\
\leq & \frac{1}{2} \int_{\Omega} \phi(\mathbf{x}) \xi^{2}\left(\mathbf{x}, t_{n}^{c}\right) d \mathbf{x}+\frac{1}{2} \int_{\Omega} \phi(\mathbf{x}) \xi^{2}\left(\mathbf{x}, t_{n-1}^{c}\right) d \mathbf{x}+3 \varepsilon \Delta t_{n}^{c}\left\|\nabla \xi\left(\mathbf{x}, t_{n}^{c}\right)\right\|^{2} \\
& +\varepsilon \Delta t_{n}^{c}\left\|\nabla \xi\left(\mathbf{x}, t_{n-1}^{c}\right)\right\|^{2}+Q \Delta t_{n}^{c}\left(\left\|\xi\left(\mathbf{x}, t_{n}^{c}\right)\right\|^{2}+\left\|\xi\left(\mathbf{x}, t_{n-1}^{c}\right)\right\|^{2}\right. \\
& \left.+\left\|\xi\left(\mathbf{x}, t_{m-1}^{p}\right)\right\|^{2}+\left\|\xi\left(\mathbf{x}, t_{m-2}^{p}\right)\right\|^{2}\right)+Q\left(\Delta t_{n}^{c}\right)^{2}\left(\|\bar{c}\|_{L^{2}\left(t_{n-1}^{c}, t_{n}^{c} ; L^{2}\right)}^{2}\right. \\
& \left.+\|c\|_{L^{2}\left(t_{n-1}^{c}, t_{n}^{c} ; H^{1}\right)}^{2}+\left\|\frac{d \bar{c}}{d t}\right\|_{L^{2}\left(t_{n-1}^{c}, t_{n}^{c} ; L^{2}\right)}^{2}+\left\|\frac{d c}{d t}\right\|_{L^{2}\left(t_{n-1}^{c}, t_{n}^{c} ; H^{1}\right)}^{2}\right) \\
& +Q \Delta t_{n}^{c}\left(\delta_{m, 1}\left(\Delta t_{p}^{1}\right)^{2}\|\mathbf{u}\|_{W_{\infty}^{1}\left(0, t_{1}^{p} ; L^{2}\right)}^{2}+\left(1-\delta_{m, 1}\right)\left(\Delta t_{p}\right)^{3}\|\mathbf{u}\|_{H^{2}\left(t_{m-2}^{p}, t_{m}^{p} ; L^{2}\right)}^{2}\right) \\
& +Q h_{c}^{2 l+2}\left(\Delta t_{n}^{c}\|c\|_{L^{\infty}\left(W_{2+\gamma}^{l+1}\right)}^{2}+\|c\|_{H^{1}\left(t_{n-1}^{c}, t_{n}^{c} ; H^{l+1}\right)}^{2}\right) \\
& +Q \Delta t_{n}^{c} h_{p}^{2 k+2}\left(\|\mathbf{u}\|_{L^{\infty}\left(H^{k+1}(\operatorname{div})\right)}^{2}+\|p\|_{L^{\infty}\left(H^{k+1}\right)}^{2}\right) .
\end{aligned}
$$

We choose $\varepsilon=|\mathbf{D}|_{\min } / 8$, sum this estimate for $n=1,2, \ldots, n^{*}$, with $n^{*} \leq N_{m}$, and cancel like terms to obtain

$$
\begin{aligned}
\int_{\Omega} \phi & (\mathbf{x}) \xi^{2}\left(\mathbf{x}, t_{n^{*}}^{c}\right) d \mathbf{x}+|\mathbf{D}|_{\min } \sum_{n=1}^{n^{*}} \Delta t_{n}^{c}\left\|\nabla \xi\left(\mathbf{x}, t_{n}^{c}\right)\right\|^{2} \\
\leq & Q \sum_{n=1}^{n^{*}} \Delta t_{n}^{c}\left\|\xi\left(\mathbf{x}, t_{n}^{c}\right)\right\|^{2}+Q\left(\Delta t_{n}^{c}\right)^{2}\left(\|\bar{c}\|_{L^{2}\left(L^{2}\right)}^{2}+\|c\|_{L^{2}\left(H^{1}\right)}^{2}\right. \\
& \left.+\left\|\frac{d \bar{c}}{d t}\right\|_{L^{2}\left(L^{2}\right)}^{2}+\left\|\frac{d c}{d t}\right\|_{L^{2}\left(H^{1}\right)}^{2}\right)+Q\left(\left(\Delta t_{p}\right)^{4}+\left(\Delta t_{p}^{1}\right)^{3}\right)\|\mathbf{u}\|_{H^{2}\left(L^{2}\right)}^{2} \\
& +Q h_{c}^{2 l+2}\left(\|c\|_{L^{\infty}\left(W_{2+\gamma}^{l+1}\right)}^{2}+\|c\|_{H^{1}\left(H^{l+1}\right)}^{2}\right) \\
& +Q h_{p}^{2 k+2}\left(\|\mathbf{u}\|_{L^{\infty}\left(H^{k+1}(\operatorname{div})\right)}^{2}+\|p\|_{L^{\infty}\left(H^{k+1}\right)}^{2}\right) .
\end{aligned}
$$

Copyright (c) by SIAM. Unauthorized reproduction of this article is prohibited. 
Here we have used the estimate (6.8) to bound the initial value $\|\xi(\mathbf{x}, 0)\|$ that has appeared on the right-hand side.

We choose $\Delta t_{n}^{c}$ small enough such that $Q \Delta t_{n}^{c}<\phi_{\min } / 2$ and apply Gronwall inequality to (5.9) to get

$$
\begin{aligned}
& \|\xi\|_{\hat{L}_{c}^{\infty}\left(0, t_{n^{*}}^{c} ; L^{2}\right)}^{2}+|\mathbf{D}|_{\min }\|\nabla \xi\|_{\hat{L}_{c}^{2}\left(0, t_{n^{*}}^{c} ; L^{2}\right)}^{2} \\
& \leq Q\left(\Delta t_{n}^{c}\right)^{2}\left(\|\bar{c}\|_{L^{2}\left(L^{2}\right)}^{2}+\|c\|_{L^{2}\left(H^{1}\right)}^{2}+\left\|\frac{d \bar{c}}{d t}\right\|_{L^{2}\left(L^{2}\right)}^{2}+\left\|\frac{d c}{d t}\right\|_{L^{2}\left(H^{1}\right)}^{2}\right) \\
& \quad+Q\left(\left(\Delta t_{p}\right)^{4}+\left(\Delta t_{p}^{1}\right)^{3}\right)\|\mathbf{u}\|_{H^{2}\left(L^{2}\right)}^{2}+Q h_{c}^{2 l+2}\left(\|c\|_{L^{\infty}\left(W_{2+\gamma}^{l+1}\right)}^{2}+\|c\|_{H^{1}\left(H^{l+1}\right)}^{2}\right) \\
& \quad+Q h_{p}^{2 k+2}\left(\|\mathbf{u}\|_{L^{\infty}\left(H^{k+1}(\mathrm{div})\right)}^{2}+\|p\|_{L^{\infty}\left(H^{k+1}\right)}^{2}\right) .
\end{aligned}
$$

Combining this estimate with (4.6) finishes the proof.

5.3. Discussion. The error estimate (5.2) contains only the $H^{1}$ norm of $c$ and its material derivative in the $\Delta t_{c}$ term. These norms are much smaller than those of the temporal derivatives $c$ due to the Lagrangian nature of the transport PDEs. Hence, larger time steps and spatial grids can be used in the ELLAM-MFEM time-stepping procedure. In other words, Theorem 5.1 theoretically justifies the mathematical and numerical strength of the ELLAM-MFEM time-stepping procedure, which was previously observed numerically $[3,17,21,30,32]$.

In the ELLAM-MFEM time-stepping procedure (3.12) and (3.14), a linear extrapolation in time is used to define the numerical velocity field $\mathbf{u}_{h}^{\mathrm{E}}\left(\mathbf{x}, t_{n}^{c}\right)$ in (3.13). A constant-in-time interpolation can be used instead:

$$
\mathbf{u}_{h}^{\mathrm{E}}\left(\mathbf{x}, t_{n}^{c}\right):=\mathbf{u}_{h}\left(\mathbf{x}, t_{m-1}^{p}\right) \quad \text { for } \quad N_{m-1}+1 \leq n \leq N_{m}, 1 \leq m \leq M .
$$

The proof in Theorem 5.1 still goes through, leading to an estimate of the form

$$
\begin{aligned}
& \left\|c_{h}-c\right\|_{\hat{L}_{c}^{\infty}\left(L^{2}\right)}+\left\|\mathbf{u}_{h}-\mathbf{u}\right\|_{\hat{L}_{p}^{\infty}(H(\mathrm{div}))}+\left\|p_{h}-p\right\|_{\hat{L}_{p}^{\infty}\left(L^{2}\right)} \\
& \quad \leq Q\left(h_{c}^{l+1}+h_{p}^{k+1}+\Delta t_{c}+\Delta t_{p}\right) .
\end{aligned}
$$

An error estimate similar to Theorem 5.1 was proved in [9] for a Galerkin FEMMFEM time-stepping procedure for problem (2.1)-(2.3) and in [13] for an MMOCMFEM time-stepping procedure. These estimates require a restrictive condition that

$$
\Delta t_{c}=o\left(h_{p}\right)
$$

In other words, these procedures are guaranteed to converge only if the Courant number tends to zero asymptotically, which is more restrictive than the CFL condition for an explicit scheme. This is especially important for the MMOC-MFEM timestepping procedure, since the strength of the MMOC scheme is really reflected in the large time steps the MMOC scheme allows.

In Theorem 5.1 the restriction (5.11) is relaxed to be

$$
\Delta t_{c}=O\left(h_{p}\right)
$$

This implies that the ELLAM-MFEM time-stepping procedure converges for any size of Courant numbers. Thus, Theorem 5.1 provides a solid theoretical foundation to show the competitiveness of the ELLAM-MFEM procedure, which was previously illustrated and observed numerically [21, 30, 32]. 
6. Auxiliary lemmas. In this section we prove several lemmas that were used in the proof of Theorem 5.1.

6.1. Estimates on tracking. In this subsection, we derive various bounds for tracking-related transformations.

Lemma 6.1. Assume that $\phi \in W_{\infty}^{1}$ and $\mathbf{u} \in L^{\infty}\left(W_{\infty}^{1}\right)$. Then the Jacobian matrix of the characteristic $\mathbf{r}\left(t ; x, t_{n-1}^{c}\right)$ is bounded in the Frobenius norm $|\cdot|_{2}$ by

$$
\left|\frac{\partial \mathbf{r}\left(t ; \mathbf{x}, t_{n-1}^{c}\right)}{\partial \mathbf{x}}-\mathbf{I}\right|_{2} \leq Q\left(t-t_{n-1}^{c}\right), \quad t \in\left[t_{n-1}^{c}, t_{n}^{c}\right]
$$

Here $Q$ depends on $\|\mathbf{u}\|_{L^{\infty}\left(W_{\infty}^{1}\right)}$ but not on discretization parameters. In addition,

$$
\operatorname{det}\left(\frac{\partial \mathbf{r}\left(t ; \mathbf{x}, t_{n-1}^{c}\right)}{\partial \mathbf{x}}\right)=1+O\left(t-t_{n-1}^{c}\right), \quad t \in\left[t_{n-1}^{c}, t_{n}^{c}\right] .
$$

Proof. Differentiating (3.15) with respect to x yields, for $t \in\left[t_{n-1}^{c}, t_{n}^{c}\right]$,

$$
\frac{\partial \mathbf{r}\left(t ; \mathbf{x}, t_{n-1}^{c}\right)}{\partial \mathbf{x}}-\mathbf{I}=\int_{t_{n-1}^{c}}^{t} \nabla\left(\frac{\mathbf{u}\left(\mathbf{r}\left(\theta ; \mathbf{x}, t_{n-1}^{c}\right), \theta\right)}{\phi\left(\mathbf{r}\left(\theta ; \mathbf{x}, t_{n-1}^{c}\right), \theta\right)}\right) \frac{\partial \mathbf{r}\left(\theta ; \mathbf{x}, t_{n-1}^{c}\right)}{\partial \mathbf{x}} d \theta .
$$

We apply Cauchy inequality to bound the Frobenius norm of $\frac{\partial \mathbf{r}}{\partial \mathbf{x}}-\mathbf{I}$ by

$$
\begin{aligned}
\left|\frac{\partial \mathbf{r}\left(t ; \mathbf{x}, t_{n-1}^{c}\right)}{\partial \mathbf{x}}-\mathbf{I}\right|_{2}^{2} & \leq\left(t-t_{n-1}^{c}\right) \int_{t_{n-1}^{c}}^{t}\left|\nabla\left(\frac{\mathbf{u}\left(\mathbf{r}\left(\theta ; \mathbf{x}, t_{n-1}^{c}\right), \theta\right)}{\phi\left(\mathbf{r}\left(\theta ; \mathbf{x}, t_{n-1}^{c}\right), \theta\right)}\right) \frac{\partial \mathbf{r}\left(\theta ; \mathbf{x}, t_{n-1}^{c}\right)}{\partial \mathbf{x}}\right|_{2}^{2} d \theta \\
& \leq\left(t-t_{n-1}^{c}\right)\left|\frac{\mathbf{u}}{\phi}\right|_{L^{\infty}\left(W_{\infty}^{1}\right)}^{2} \int_{t_{n-1}^{c}}^{t}\left|\frac{\partial \mathbf{r}\left(\theta ; \mathbf{x}, t_{n-1}^{c}\right)}{\partial \mathbf{x}}\right|_{2}^{2} d \theta \\
& \leq Q\left(t-t_{n-1}^{c}\right)^{2}+Q\left(t-t_{n-1}^{c}\right) \int_{t_{n-1}^{c}}^{t}\left|\frac{\partial \mathbf{r}\left(\theta ; \mathbf{x}, t_{n-1}^{c}\right)}{\partial \mathbf{x}}-\mathbf{I}\right|_{2}^{2} d \theta
\end{aligned}
$$

Applying Gronwall inequality finishes the proof of (6.1), which directly gives (6.2).

LEmma 6.2. Under the assumptions of Theorem 5.1,

$$
h_{p}^{-1} h_{c}^{l+1}\left\|c_{0}\right\|_{H^{l+1}} \leq 1
$$

and

$$
\frac{\Delta t_{c}}{h_{p}} \leq Q_{2}, \quad \frac{\Delta t_{p}}{h_{p}^{1 / 2}} \leq Q_{2}, \quad \frac{\Delta t_{p}^{1}}{h_{p}^{2 / 3}} \leq Q_{2},
$$

we have the following estimate for the difference $\tilde{\mathbf{x}}_{h}-\tilde{\mathbf{x}}$ :

$$
\left\|\tilde{\mathbf{x}}_{h}-\tilde{\mathbf{x}}\right\|_{L^{q}} \leq Q_{3} \Delta t_{n}^{c} h_{p}^{\frac{2}{q}} .
$$

Copyright (c) by SIAM. Unauthorized reproduction of this article is prohibited. 
Proof. We use (3.15) and (3.18) and the estimates (4.3) and (5.3) to bound $\left\|\tilde{\mathbf{x}}_{h}-\tilde{\mathbf{x}}\right\|_{L^{q}}$ for $2 \leq q \leq+\infty$ by

$$
\begin{aligned}
\left\|\tilde{\mathbf{x}}_{h}-\tilde{\mathbf{x}}\right\|_{L^{q}}= & \left\|\frac{\mathbf{u}_{h}^{\mathrm{E}}\left(\mathbf{x}, t_{n-1}^{c}\right)}{\phi(\mathbf{x})} \Delta t_{n}^{c}-\int_{t_{n-1}^{c}}^{t_{n}^{c}} \frac{\mathbf{u}\left(\mathbf{r}\left(\theta ; \mathbf{x}, t_{n-1}^{c}\right), \theta\right)}{\phi\left(\mathbf{r}\left(\theta ; \mathbf{x}, t_{n-1}^{c}\right)\right)} d \theta\right\|_{L^{q}} \\
= & \| \Delta t_{n}^{c}\left(\frac{\mathbf{u}_{h}^{\mathrm{E}}\left(\mathbf{x}, t_{n-1}^{c}\right)-\mathbf{u}^{E}\left(\mathbf{x}, t_{n-1}^{c}\right)}{\phi(\mathbf{x})}+\frac{\mathbf{u}^{E}\left(\mathbf{x}, t_{n-1}^{c}\right)-\mathbf{u}\left(\mathbf{x}, t_{n-1}^{c}\right)}{\phi(\mathbf{x})}\right) \\
& \quad-\int_{t_{n-1}^{c}}^{t_{n}^{c}}\left(\frac{\mathbf{u}\left(\mathbf{r}\left(\theta ; \mathbf{x}, t_{n-1}^{c}\right), \theta\right)}{\phi\left(\mathbf{r}\left(\theta ; \mathbf{x}, t_{n-1}^{c}\right)\right)}-\frac{\mathbf{u}\left(\mathbf{x}, t_{n-1}^{c}\right)}{\phi(\mathbf{x})}\right) d \theta \|_{L^{q}} \\
\leq & 3 \phi_{m i n}^{-1} \Delta t_{n}^{c}\left(\left\|\mathbf{u}_{h}-\mathbf{u}\right\|_{\hat{L}_{p}^{\infty}\left(t_{m-2}^{p}, t_{m-1}^{p} ; L^{q}\right)}+\delta_{m, 1} \Delta t_{p}^{1}\|\mathbf{u}\|_{W_{1}^{\infty}\left(t_{0}^{p}, t_{1}^{p} ; L^{q}\right)}\right. \\
& \left.+\left(1-\delta_{m, 1}\right)\left(\Delta t_{p}\right)^{3 / 2}\|\mathbf{u}\|_{H^{2}\left(t_{m-2}^{p}, t_{m}^{p} ; L^{q}\right)}+Q \Delta t_{n}^{c}\right) .
\end{aligned}
$$

Here $Q$ depends on $\left\|\frac{d \mathbf{u}}{d t}\right\|_{L^{\infty}\left(L^{\infty}\right)}$ and $\|\mathbf{u}\|_{L^{\infty}\left(L^{\infty}\right)}$ but not on discretization parameters.

We combine (4.1), (4.6), and (5.3) to conclude that

$$
\begin{aligned}
\|\xi(\mathbf{x}, 0)\| & \leq\left\|c_{h}(\mathbf{x}, 0)-\Pi c_{0}(\mathbf{x})\right\| \\
& \leq\left\|c_{h}(\mathbf{x}, 0)-c_{0}(\mathbf{x})\right\|+\left\|\Pi c_{0}(\mathbf{x})-c_{0}(\mathbf{x})\right\| \leq 2 A_{1} h_{c}^{l+1}\left\|c_{0}\right\|_{H^{l+1}}
\end{aligned}
$$

and

$$
\begin{aligned}
& \left\|\left(\mathbf{u}_{h}-\Pi \mathbf{u}\right)(\mathbf{x}, 0)\right\|_{L^{q}} \\
& \quad \leq K_{2} h_{p}^{-\left(1-\frac{2}{q}\right)}\left\|\left(\mathbf{u}_{h}-\Pi \mathbf{u}\right)(\mathbf{x}, 0)\right\| \leq K_{2} Q_{1} h_{p}^{-\left(1-\frac{2}{q}\right)}\left\|c_{h}(\mathbf{x}, 0)-c_{o}(\mathbf{x})\right\| \\
& \quad \leq A_{1} K_{2} Q_{1} h_{p}^{-\left(1-\frac{2}{q}\right)} h_{c}^{l+1}\left\|c_{o}\right\|_{H^{l+1}} \leq A_{1} K_{2} Q_{1} h_{p}^{\frac{2}{q}},
\end{aligned}
$$

provided that condition (6.4) is true.

By (4.4) and (5.1), the estimate (6.7) reduces to the following for $n=1,2, \ldots, N_{1}$ :

$$
\begin{aligned}
\left\|\tilde{\mathbf{x}}_{h}-\tilde{\mathbf{x}}\right\|_{L^{q}} \leq & 3 \phi_{\min }^{-1} \Delta t_{n}^{c}\left(\left\|\left(\mathbf{u}_{h}-\mathbf{u}\right)(\mathbf{x}, 0)\right\|_{L^{q}}+\Delta t_{p}^{1}\|\mathbf{u}\|_{W_{1}^{\infty}\left(t_{0}^{p}, t_{1}^{p} ; L^{q}\right)}+Q \Delta t_{n}^{c}\right) \\
\leq & 3 \phi_{\min }^{-1} \Delta t_{n}^{c}\left(A_{2} K_{2} h_{p}^{k+\frac{2}{q}}\left(\|\mathbf{u}\|_{L^{\infty}\left(H^{k+1}(\operatorname{div})\right)}+\|p\|_{L^{\infty}\left(H^{k+1}\right)}\right)\right. \\
& \left.\quad+A_{1} K_{2} Q_{1} h_{p}^{\frac{2}{q}}+\Delta t_{p}^{1}\|\mathbf{u}\|_{W_{1}^{\infty}\left(t_{0}^{p}, t_{1}^{p} ; L^{q}\right)}+Q \Delta t_{n}^{c}\right) \\
\leq & Q_{4} \Delta t_{n}^{c} h_{p}^{\frac{2}{q}}
\end{aligned}
$$

For $n=N_{m-1}+1,2, \ldots, N_{m}$, we use (4.4), (5.1), (5.2), and (6.5) to bound (6.7) as follows:

$$
\begin{aligned}
\| \tilde{\mathbf{x}}_{h} & -\tilde{\mathbf{x}} \|_{L^{q}} \\
\leq & 3 \phi_{\min }^{-1} \Delta t_{n}^{c}\left[\left\|\mathbf{u}_{h}-\mathbf{u}\right\|_{\hat{L}_{p}^{\infty}\left(t_{m-2}^{p}, t_{m-1}^{p} ; L^{q}\right)}+\delta_{m, 1} \Delta t_{p}^{1}\|\mathbf{u}\|_{W_{1}^{\infty}\left(t_{0}^{p}, t_{1}^{p} ; L^{q}\right)}\right. \\
& \left.+\left(1-\delta_{m, 1}\right)\left(\Delta t_{p}\right)^{3 / 2}\|\mathbf{u}\|_{H^{2}\left(t_{m-2}^{p}, t_{m}^{p} ; L^{q}\right)}+Q \Delta t_{n}^{c}\right] \\
\leq & 3 \phi_{\min }^{-1} \Delta t_{n}^{c} h_{p}^{\frac{2}{q}}\left[\left(A_{2}+Q^{*}\right) K_{2} h_{p}^{k}\left(\|\mathbf{u}\|_{L^{\infty}\left(H^{k+1}(\mathrm{div})\right)}+\|p\|_{L^{\infty}\left(H^{k+1}\right)}\right)\right. \\
& +Q^{*} Q_{2}\left(\|\bar{c}\|_{L^{2}\left(L^{2}\right)}+\|c\|_{L^{2}\left(H^{1}\right)}+\left\|\frac{d \bar{c}}{d \tau}\right\|_{L^{2}\left(L^{2}\right)}+\left\|\frac{d c}{d \tau}\right\|_{L^{2}\left(H^{1}\right)}\right) \\
& \left.+Q^{*} Q_{2}\|\mathbf{u}\|_{H^{2}\left(L^{2}\right)}+Q^{*}\right] \\
\leq & Q_{3} \Delta t_{n}^{c} h_{p}^{\frac{2}{q}} .
\end{aligned}
$$

Copyright $@$ by SIAM. Unauthorized reproduction of this article is prohibited. 
6.2. Miscellaneous estimates. In this subsection we prove the estimates (5.5), (5.6), and (5.7) for the fifth, the first, and the second and seventh terms on the righthand side of (5.4), respectively.

Lemma 6.3. Under the conditions of Theorem 5.1, estimate (5.5) holds for $n=$ $N_{m-1}+1, \ldots, N_{m}$.

Proof. It is straightforward to see that [8]

$$
|\mathbf{D}(\mathbf{x}, \mathbf{u})-\mathbf{D}(\mathbf{x}, \mathbf{v})| \leq Q_{4}|\mathbf{u}-\mathbf{v}|
$$

With this we bound the left-hand side of (5.5) by

$$
\begin{aligned}
\mid \Delta & t_{n}^{c} \int_{\Omega} \nabla \xi\left(\mathbf{x}, t_{n}^{c}\right) \cdot\left(\mathbf{D}\left(\mathbf{x}, \mathbf{u}_{h}^{E}\left(\mathbf{x}, t_{n}^{c}\right)\right)-\mathbf{D}\left(\mathbf{x}, \mathbf{u}\left(\mathbf{x}, t_{n}^{c}\right)\right)\right) \nabla \Pi c\left(\mathbf{x}, t_{n}^{c}\right) d \mathbf{x} \mid \\
\leq & Q_{4} \Delta t_{n}^{c}\left\|\nabla \xi\left(\mathbf{x}, t_{n}^{c}\right)\right\|\left\|\mathbf{u}_{h}^{E}\left(\mathbf{x}, t_{n}^{c}\right)-\mathbf{u}\left(\mathbf{x}, t_{n}^{c}\right)\right\|\left\|\Pi c\left(\mathbf{x}, t_{n}^{c}\right)\right\|_{W_{\infty}^{1}} \\
\leq & K_{3} Q_{4} \Delta t_{n}^{c}\left\|\nabla \xi\left(\mathbf{x}, t_{n}^{c}\right)\right\|\left(\left\|\mathbf{u}_{h}^{E}\left(\mathbf{x}, t_{n}^{c}\right)-(\Pi \mathbf{u})^{E}\left(\mathbf{x}, t_{n}^{c}\right)\right\|\right. \\
& \left.+\left\|(\Pi \mathbf{u})^{E}\left(\mathbf{x}, t_{n}^{c}\right)-\mathbf{u}^{E}\left(\mathbf{x}, t_{n}^{c}\right)\right\|+\left\|\mathbf{u}^{E}\left(\mathbf{x}, t_{n}^{c}\right)-\mathbf{u}\left(\mathbf{x}, t_{n}^{c}\right)\right\|\right),
\end{aligned}
$$

where at the last " $\leq$ " sign we have used (4.7).

The last term in the bracket is bounded in (4.12). We use the estimate (4.9) to bound the second term in the bracket to get

$$
\begin{aligned}
& \left\|(\Pi \mathbf{u})^{E}\left(\mathbf{x}, t_{n}^{c}\right)-\mathbf{u}^{E}\left(\mathbf{x}, t_{n}^{c}\right)\right\| \\
& \quad \leq Q\left(\left\|\Pi \mathbf{u}\left(\mathbf{x}, t_{m-2}^{p}\right)-\mathbf{u}\left(\mathbf{x}, t_{m-2}^{p}\right)\right\|+\left\|\Pi \mathbf{u}\left(\mathbf{x}, t_{m-1}^{p}\right)-\mathbf{u}\left(\mathbf{x}, t_{m-1}^{p}\right)\right\|\right) \\
& \quad \leq Q h_{p}^{k+1}\left(\|\mathbf{u}\|_{L^{\infty}\left(H^{k+1}(\operatorname{div})\right)}+\|p\|_{L^{\infty}\left(H^{k+1}\right)}\right) .
\end{aligned}
$$

We use (4.6) and (5.3) to bound the first term in the bracket of (6.12) by

$$
\begin{aligned}
& \left\|\mathbf{u}_{h}^{E}\left(\mathbf{x}, t_{n}^{c}\right)-(\Pi \mathbf{u})^{E}\left(\mathbf{x}, t_{n}^{c}\right)\right\| \\
& \quad \leq Q\left(\left\|\mathbf{u}_{h}\left(\mathbf{x}, t_{m-2}^{p}\right)-\Pi \mathbf{u}\left(\mathbf{x}, t_{m-2}^{p}\right)\right\|+\left\|\mathbf{u}_{h}\left(\mathbf{x}, t_{m-1}^{p}\right)-\Pi \mathbf{u}\left(\mathbf{x}, t_{m-1}^{p}\right)\right\|\right) \\
& \quad \leq Q\left(\left\|c_{h}\left(\mathbf{x}, t_{m-2}^{p}\right)-c\left(\mathbf{x}, t_{m-2}^{p}\right)\right\|+\left\|c_{h}\left(\mathbf{x}, t_{m-1}^{p}\right)-c\left(\mathbf{x}, t_{m-1}^{p}\right)\right\|\right) \\
& \quad \leq Q\left(\left\|\xi\left(\mathbf{x}, t_{m-2}^{p}\right)\right\|+\left\|\xi\left(\mathbf{x}, t_{m-1}^{p}\right)\right\|\right)+Q h_{c}^{l+1}\|c\|_{L^{\infty}\left(H^{l+1}\right)} .
\end{aligned}
$$

We combine these two estimates with (4.12) to complete the proof.

Lemma 6.4. Under the conditions of Theorem 5.1, estimate (5.6) holds for $n=$ $N_{m-1}+1, \ldots, N_{m}$.

Proof. The first term on the right-hand side of (5.4) can be written as

$$
\begin{aligned}
\int_{\Omega} \phi(\mathbf{x}) \xi\left(\mathbf{x}, t_{n-1}^{c}\right) \xi\left(\mathbf{x}, t_{n-1}^{c,+}\right) d \mathbf{x}= & \int_{\Omega} \phi(\mathbf{x}) \xi\left(\mathbf{x}, t_{n-1}^{c}\right) \xi\left(\tilde{\mathbf{x}}_{h}, t_{n}^{c}\right) d \mathbf{x} \\
= & \int_{\Omega} \phi(\mathbf{x}) \xi\left(\mathbf{x}, t_{n-1}^{c}\right) \xi\left(\tilde{\mathbf{x}}, t_{n}^{c}\right) d \mathbf{x} \\
& +\int_{\Omega} \phi(\mathbf{x}) \xi\left(\mathbf{x}, t_{n-1}^{c}\right)\left(\xi\left(\tilde{\mathbf{x}}_{h}, t_{n}^{c}\right)-\xi\left(\tilde{\mathbf{x}}, t_{n}^{c}\right)\right) d \mathbf{x} .
\end{aligned}
$$

Copyright (c) by SIAM. Unauthorized reproduction of this article is prohibited. 
We use estimate (6.2) to bound the first term on the right-hand side of (6.13)

$$
\begin{aligned}
&\left|\int_{\Omega} \phi(\mathbf{x}) \xi\left(\mathbf{x}, t_{n-1}^{c}\right) \xi\left(\tilde{\mathbf{x}}, t_{n}^{c}\right) d \mathbf{x}\right| \\
& \leq \frac{1}{2} \int_{\Omega} \phi(\mathbf{x}) \xi^{2}\left(\mathbf{x}, t_{n-1}^{c}\right) d \mathbf{x}+\frac{1}{2} \int_{\Omega} \phi(\mathbf{x}) \xi^{2}\left(\tilde{\mathbf{x}}, t_{n}^{c}\right) d \mathbf{x} \\
&= \frac{1}{2} \int_{\Omega} \phi(\mathbf{x}) \xi^{2}\left(\mathbf{x}, t_{n-1}^{c}\right) d \mathbf{x}+\frac{1}{2} \int_{\Omega} \phi(\tilde{\mathbf{x}}) \xi^{2}\left(\tilde{\mathbf{x}}, t_{n}^{c}\right) \operatorname{det}\left(\frac{\partial \mathbf{x}}{\partial \tilde{\mathbf{x}}}\right) d \tilde{\mathbf{x}} \\
&+\frac{1}{2} \int_{\Omega}(\phi(\mathbf{x})-\phi(\tilde{\mathbf{x}})) \xi^{2}\left(\tilde{\mathbf{x}}, t_{n}^{c}\right) \operatorname{det}\left(\frac{\partial \mathbf{x}}{\partial \tilde{\mathbf{x}}}\right) d \tilde{\mathbf{x}} \\
& \leq \frac{1}{2} \int_{\Omega} \phi(\mathbf{x}) \xi^{2}\left(\mathbf{x}, t_{n-1}^{c}\right) d \mathbf{x}+\frac{1}{2} \int_{\Omega} \phi(\mathbf{x}) \xi^{2}\left(\mathbf{x}, t_{n}^{c}\right) d \mathbf{x}+Q \Delta t_{n}^{c}\left\|\xi\left(\mathbf{x}, t_{n}^{c}\right)\right\|^{2} .
\end{aligned}
$$

Here we have rewritten $\tilde{\mathbf{x}}$ as $\mathbf{x}$ in the second term.

We use (4.2), (6.6), and the relationship

$$
\xi\left(\tilde{\mathbf{x}}_{h}, t_{n}^{c}\right)-\xi\left(\tilde{\mathbf{x}}, t_{n}^{c}\right)=\left(\tilde{\mathbf{x}}_{h}-\tilde{\mathbf{x}}\right) \cdot \int_{0}^{1} \nabla \xi\left(\tilde{\mathbf{x}}+s\left(\tilde{\mathbf{x}}_{h}-\tilde{\mathbf{x}}\right), t_{n}^{c}\right) d s,
$$

to bound the second term on the right-hand side of (6.13) by

$$
\begin{aligned}
& \left|\int_{\Omega} \phi(\mathbf{x}) \xi\left(\mathbf{x}, t_{n-1}^{c}\right)\left(\xi\left(\tilde{\mathbf{x}}_{h}, t_{n}^{c}\right)-\xi\left(\tilde{\mathbf{x}}, t_{n}^{c}\right)\right) d \mathbf{x}\right| \\
& \quad \leq Q\left\|\xi\left(\mathbf{x}, t_{n-1}^{c}\right)\right\|_{L^{\infty}}\left\|\tilde{\mathbf{x}}_{h}-\tilde{\mathbf{x}}\right\|\left(\int_{0}^{1} \int_{\Omega}\left|\nabla \xi\left(\tilde{\mathbf{x}}+s\left(\tilde{\mathbf{x}}_{h}-\tilde{\mathbf{x}}\right), t_{n}^{c}\right)\right|^{2} d \mathbf{x} d s\right)^{1 / 2} \\
& \quad \leq K_{1} Q\left|\ln h_{c}\right|^{1 / 2}\left\|\xi\left(\mathbf{x}, t_{n-1}^{c}\right)\right\|_{H^{1}}\left\|\tilde{\mathbf{x}}_{h}-\tilde{\mathbf{x}}\right\|\left\|\nabla \xi\left(\mathbf{x}, t_{n-1}^{c}\right)\right\| \\
& \quad \leq \varepsilon \Delta t_{n}^{c}\left(\left\|\nabla \xi\left(\mathbf{x}, t_{n}^{c}\right)\right\|^{2}+\left\|\nabla \xi\left(\mathbf{x}, t_{n-1}^{c}\right)\right\|^{2}+\left\|\xi\left(\mathbf{x}, t_{n-1}^{c}\right)\right\|^{2}\right),
\end{aligned}
$$

provided that

$$
2 K_{1} Q Q_{3} h_{p}^{2 q}\left|\ln h_{c}\right|^{1 / 2} \leq \varepsilon, \quad 2 K_{1} Q Q_{4} h_{p}^{2 q}\left|\ln h_{c}\right|^{1 / 2} \leq \varepsilon .
$$

LEMma 6.5. Under the conditions of Theorem 5.1, estimate (5.7) holds for $n=$ $N_{m-1}+1, \ldots, N_{m}$.

Proof. We decompose the second and seventh terms on the right-hand side of (5.4) as follows:

$$
\begin{aligned}
\int_{\Omega} \phi(\mathbf{x}) \eta\left(\mathbf{x}, t_{n}^{c}\right) \xi\left(\mathbf{x}, t_{n}^{c}\right) d \mathbf{x}-\int_{\Omega} \phi(\mathbf{x}) \eta\left(\mathbf{x}, t_{n-1}^{c}\right) \xi\left(\mathbf{x}, t_{n-1}^{c,+}\right) d \mathbf{x} \\
=\int_{\Omega} \phi(\mathbf{x})\left[\int_{t_{n-1}^{c}}^{t_{n}^{c}} \frac{\partial \eta(\mathbf{x}, t)}{\partial t} d t\right] \xi\left(\mathbf{x}, t_{n}^{c}\right) d \mathbf{x} \\
\quad-\int_{\Omega} \phi(\mathbf{x}) \eta\left(\mathbf{x}, t_{n-1}^{c}\right)\left(\xi\left(\tilde{\mathbf{x}}_{h}, t_{n}^{c}\right)-\xi\left(\tilde{\mathbf{x}}, t_{n}^{c}\right)\right) d \mathbf{x} \\
\quad-\int_{\Omega} \phi(\mathbf{x}) \eta\left(\mathbf{x}, t_{n-1}^{c}\right)\left(\xi\left(\tilde{\mathbf{x}}, t_{n}^{c}\right)-\xi\left(\mathbf{x}, t_{n}^{c}\right)\right) d \mathbf{x} .
\end{aligned}
$$

The first term on the right-hand side of (6.18) is bounded by

$$
\begin{aligned}
& \left|\int_{\Omega} \phi(\mathbf{x})\left[\int_{t_{n-1}^{c}}^{t_{n}^{c}} \frac{\partial \eta(\mathbf{x}, t)}{\partial t} d t\right] \xi\left(\mathbf{x}, t_{n}^{c}\right) d \mathbf{x}\right| \\
& \quad \leq Q\left(\Delta t_{n}^{c}\right)^{1 / 2}\left\|\xi\left(\mathbf{x}, t_{n}^{c}\right)\right\|\|\eta\|_{H^{1}\left(t_{n-1}^{c}, t_{n}^{c} ; L^{2}\right)} \\
& \quad \leq Q \Delta t_{n}^{c}\left\|\xi\left(\mathbf{x}, t_{n}^{c}\right)\right\|^{2}+A_{1}^{2} h_{c}^{2 l+2}\|c\|_{H^{1}\left(t_{n-1}^{c}, t_{n}^{c} ; H^{l+1}\right)}
\end{aligned}
$$

Copyright $@$ by SIAM. Unauthorized reproduction of this article is prohibited. 
We use (3.15) to bound the third term on the right-hand side of (6.18)

$$
\begin{aligned}
& \left|\int_{\Omega} \phi(\mathbf{x}) \eta\left(\mathbf{x}, t_{n-1}^{c}\right)\left(\xi\left(\tilde{\mathbf{x}}, t_{n}^{c}\right)-\xi\left(\mathbf{x}, t_{n}^{c}\right)\right) d \mathbf{x}\right| \\
& \quad=\left|\int_{\Omega} \phi(\mathbf{x}) \eta\left(\mathbf{x}, t_{n-1}^{c}\right)(\tilde{\mathbf{x}}-\mathbf{x}) \cdot \int_{0}^{1} \nabla \xi\left(\mathbf{x}+s(\tilde{\mathbf{x}}-\mathbf{x}), t_{n}^{c}\right) d \mathbf{x}\right| \\
& \quad \leq Q \Delta t_{n}^{c}\left\|\nabla \xi\left(\mathbf{x}, t_{n}\right)\right\|\left\|\eta\left(\mathbf{x}, t_{n-1}^{c}\right)\right\| \\
& \quad \leq \varepsilon \Delta t_{n}^{c}\left\|\nabla \xi\left(\mathbf{x}, t_{n}^{c}\right)\right\|^{2}+Q \Delta t_{n}^{c} h_{c}^{2 l+2}\|c\|_{L^{\infty}\left(H^{l+1}\right)}^{2} .
\end{aligned}
$$

We use the estimate (6.6) with $q=2(2+\gamma) / \gamma$ and the generalized Hölder inequality to bound the second term on the right-hand side of (6.18) in a similar manner to the estimate of (6.16) as follows:

$$
\begin{aligned}
& \left|\int_{\Omega} \phi(\mathbf{x}) \eta\left(\mathbf{x}, t_{n-1}^{c}\right)\left(\xi\left(\tilde{\mathbf{x}}_{h}, t_{n}^{c}\right)-\xi\left(\tilde{\mathbf{x}}, t_{n}^{c}\right)\right) d \mathbf{x}\right| \\
& \quad \leq Q\left\|\tilde{\mathbf{x}}_{h}-\tilde{\mathbf{x}}\right\|_{L^{\frac{2(2+\gamma}{\gamma}}}\left\|\eta\left(\mathbf{x}, t_{n-1}^{c}\right)\right\|_{L^{2+\gamma}}\left\|\nabla \xi\left(\mathbf{x}, t_{n}^{c}\right)\right\| \\
& \quad \leq \varepsilon \Delta t_{n}^{c}\left\|\nabla \xi\left(\mathbf{x}, t_{n}^{c}\right)\right\|^{2}+Q \Delta t_{n}^{c} h_{c}^{2 l+2}\|c\|_{L^{\infty}\left(W_{2+\gamma}^{l+1}\right)}^{2}
\end{aligned}
$$

provided that

$$
Q Q_{3} h_{p}^{\frac{\gamma}{2+\gamma}} \leq \varepsilon, \quad Q Q_{4} h_{p}^{\frac{\gamma}{2+\gamma}} \leq \varepsilon .
$$

Acknowledgments. The author expresses his sincere thanks to the referees for their very helpful comments and suggestions, which greatly improved the quality of this paper.

\section{REFERENCES}

[1] T. Arbogast and M.F. Wheeler, A characteristics-mixed finite element method for advection-dominated transport problems, SIAM J. Numer. Anal., 32 (1995), pp. 404-424.

[2] J. Bear, Dynamics of Fluids in Porous Materials, Elsevier, New York, 1972.

[3] P.J. Binning AND M.A. CELIA, A finite volume Eulerian-Lagrangian localized adjoint method for solution of the contaminant transport equations in two-dimensional multi-phase flow systems, Water Resour. Res., 32 (1996), pp. 103-114.

[4] F. BrezzI, On the existence, uniqueness and approximation of saddle-point problems arising from Lagrangian multipliers, RAIRO Anal. Numer., 8 (1974), pp. 129-151.

[5] F. Brezzi And M. Fortin, Mixed and Hybrid Finite Element Methods, Springer-Verlag, New York, 1991

[6] M.A. Celia, T.F. Russell, I. Herrera, and R.E. Ewing, An Eulerian-Lagrangian localized adjoint method for the advection-diffusion equation, Adv. Wat. Resour., 13 (1990), pp. 187206.

[7] P.G. Ciarlet, The Finite Element Method for Elliptic Problems, North-Holland, Amsterdam, 1978.

[8] J. Douglas, JR., R.E. Ewing, And M.F. Wheeler, The approximation of the pressure by a mixed method in the simulation of miscible displacement, RAIRO Anal. Numer., 17 (1983), pp. 17-33.

[9] J. Douglas, JR., R.E. Ewing, And M.F. Wheeler, A time-discretization procedure for a mixed finite element approximation of miscible displacement in porous media, RAIRO Anal. Numer., 17 (1983), pp. 249-265.

[10] J. Douglas, JR., And T.F. Russell, Numerical methods for convection-dominated diffusion problems based on combining the method of characteristics with finite element or finite difference procedures, SIAM J. Numer. Anal., 19 (1982), pp. 871-885.

[11] R.E. EwIng, ED., The Mathematics of Reservoir Simulation, SIAM, Philadelphia, 1984.

Copyright (c) by SIAM. Unauthorized reproduction of this article is prohibited. 
[12] R.E. Ewing, T.F. Russell, And M.F. Wheeler, Simulation of miscible displacement using mixed methods and a modified method of characteristics, SPE 12241, (1983), pp. 71-81.

[13] R.E. Ewing, T.F. Russell, And M.F. WheEler, Convergence analysis of an approximation of miscible displacement in porous media by mixed finite elements and a modified method of characteristics, Comput. Methods Appl. Mech. Engrg., 47 (1984), pp. 73-92.

[14] R.E. EWING AND H. WANG, Eulerian-Lagrangian localized adjoint methods for linear advection or advection-reaction equations and their convergence analysis, Comput. Mech., 12 (1993), pp. $97-121$.

[15] R.E. Ewing AND H. WANG, An optimal-order error estimate to Eulerian-Lagrangian localized adjoint methods for variable-coefficient advection-reaction problems, SIAM J. Numer. Anal., 33 (1996), pp. 318-348.

[16] R.E. EWING AND H. WANG, A summary of numerical methods for time-dependent advectiondominated partial differential equations, J. Comput. Appl. Math., 128 (2001), pp. 423-445.

[17] C.I. Heberton, T.F. Russell, L.F. Konikow, and G.Z. Hornberger, Three-dimensional finite-volume ELLAM implementation, in Computational Methods in Water Resources XIII, L. Bently et al. eds., Balkema, Rotterdam, pp. 603-610.

[18] K.W. Morton, Numerical Solution of Convection-Diffusion Problems, Chapman \& Hall, London, 1996.

[19] R. Rannacher and R. Scott, Some optimal error estimate for piecewise linear finite element approximations, Math. Comp., 38 (1982), pp. 437-445.

[20] P.-A. Raviart and J.-M. Thomas, A Mixed Finite Element Method for Second Order Elliptic Problems, Lecture Notes in Math. 606, Galligani and Magenes, eds., Springer-Verlag, Berlin, 1977, pp. 292-315.

[21] T.F. Russell And M.A. Celia, An overview of research on Eulerian-Lagrangian localized adjoint methods (ELLAM), Adv. Wat. Resour., 25 (2002), pp. 1215-1231.

[22] T.F. Russell AND M.F. Wheeler, Finite element and finite difference methods for continuous flows in porous media, in The Mathematics of Reservoir Simulation, R. E. Ewing, ed., SIAM, Philadelphia, 1984, pp. 35-106.

[23] V. Thomée, Galerkin Finite Element Methods for Parabolic Problems, Lecture Notes in Math. 1054, Springer-Verlag, New York, 1984.

[24] H. WANG, A family of ELLAM Schemes for advection-diffusion-reaction equations and their convergence analyses, Numer. Methods Partial Differential Equations, 14 (1998), pp. 739780 .

[25] H. WANG, An optimal-order error estimate for an ELLAM scheme for two-dimensional linear advection-diffusion equations, SIAM J. Numer. Anal., 37 (2000), pp. 1338-1368.

[26] H. Wang, H.K. Dahle, R.E. Ewing, M.S. Espedal, R.C. Sharpley, and S. Man, An ELLAM scheme for advection-diffusion equations in two dimensions, SIAM J. Sci. Comput., 20 (1999), pp. 2160-2194.

[27] H. WANG AND R.E. EwING, Optimal-order convergence rates for Eulerian-Lagrangian localized adjoint methods for reactive transport and contamination in groundwater, Numer. Methods Partial Differential Equations, 11 (1995), pp. 1-31.

[28] H. Wang, R.E. Ewing, G. Qin, S.L. Lyons, M. Al-Lawatia, and S. Man, A family of Eulerian-Lagrangian localized adjoint methods for multi-dimensional advection-reaction equations, J. Comput. Phys., 152 (1999), pp. 120-163.

[29] H. WAng, R.E. Ewing, AND T.F. Russell, Eulerian-Lagrangian localized methods for convection-diffusion equations and their convergence analysis, IMA J. Numer. Anal., 15 (1995), pp. 405-459.

[30] H. Wang, D. Liang, R.E. Ewing, S.L. Lyons, And G. QIn, An approximation to miscible fluid flows in porous media with point sources and sinks by an Eulerian-Lagrangian localized adjoint method and mixed finite element methods, SIAM J. Sci. Comput., 22 (2000), pp. 561-581.

[31] K. WANG, A uniformly optimal-order error estimate of an ELLAM scheme for unsteady-state advection-diffusion equations, Internat. J. Numer. Anal. and Modeling, 5 (2008), pp. 286302.

[32] K. Wang, H. Wang, AND M. Al-LaWATia, An Eulerian-Lagrangian discontinuous Galerkin method for transient advection-diffusion equations, Numer. Methods Partial Differential Equations, 23 (2007), pp. 1343-1367.

[33] M.F. WheELER, A priori $L_{2}$ error estimates for Galerkin approximations to parabolic partial differential equations, SIAM J. Numer. Anal., 10 (1973), pp. 723-759.

Copyright (c) by SIAM. Unauthorized reproduction of this article is prohibited. 